\title{
MARX' UND ENGELS' AUSEINANDERSETZUNG MIT DARWIN
}

\section{ZUR DIFFERENZ ZWISCHEN MARX UND ENGELS}

Seit der vollständigen Veröffentlichung der Marxschen Frühschriften ist es gesichertes Ergebnis der Forschung, daß der philosophische Ansatz des jungen Marx fundamental von dem verschieden ist, was der späte Engels im weltanschaulichen System des "Anti-Dühring” ( $1877 / 78$ ) als Marxismus formuliert hat. Iring Fetscher hat diesen Gegensatz vorläufig abschließend dargelegt. ${ }^{1}$ Chronologisch differenziert wurde dieses Bild durch Hermann Bollnow, der gezeigt hat, daß sich Engels, der bei der „Deutschen Ideologie” (1 845) zur völligen Identität der Auffassung mit Marx gelangt war, bereits in seinen "Grundsätzen des Kommunismus" (1847) in Richtung auf den "Anti-Dühring" von Marx entfernt hat - wenn auch erst in einem Maße, daß nur die feinsten interpretatorischen Mittel dem auf die Spur kommen können. ${ }^{2}$ Alfred Schmidt hat dann nachgewiesen, daß der Kritik der politischen Ökonomie des mittleren und späten Marx ein nicht-ontologischer Materialismus zugrundeliegt, während Engels' „Naturdialektik" zwar um die Gefahr der Ontologie weiß, jedoch nur unzureichend gegen sie gesichert ist und in den entscheidenden Aussagen in einen metaphysischen Materialismus abgleitet. ${ }^{3}$ Die Differenz zwischen Marx und Engels ist also bisher in den prinzipiell-philosophischen Fragen untersucht worden. Wir wollen an Hand der Auseinandersetzung, die Marx und Engels mit Darwin geführt haben, die Differenz auf einem anderen Felde darstellen und dabei zu zeigen versuchen, wie sich die unterschiedlichen philosophischen Ansätze auf ein Randproblem - und um das handelt es sich hier natürlich -

\footnotetext{
${ }^{1}$ I. Fetscher, Von der Philosophie des Proletariats zur proletarischen Weltanschauung, in: Marxismusstudien, II, Tübingen 1957, S. 26-5 I.

${ }^{2}$ H. Bollnow, Engels' Auffassung von Revolution und Entwicklung in seinen "Grundsätzen des Kommunismus" (1847), in: Marxismusstudien, I, Tübingen 1954, S. 77-144. ${ }^{3}$ A. Schmidt, Der Begriff der Natur in der Lehre von Marx, Frankfurt 1962 [Frankfurter Beiträge zur Soziologie, I I], bes. S. I 2 ff., 4 I ff.
} 
ausgewirkt haben. Die bisherige Literatur über die Beziehung von Marx und Engels zu Darwin, ${ }^{\mathbf{1}}$ auch in anderer Hinsicht ungenügend, ist nicht bis zu dem Punkt vorgedrungen, an dem eine Differenz zwischen Marx und Engels sichtbar wird.

Die Untersuchung verfolgt zugleich noch einen anderen Zweck. Mit der Herausarbeitung der ursprünglichen Gestalt des historischen Materialismus ist der späte Marx für die Forschung zum Problem geworden. Generell kann das zwar nach Alfred Schmidts Untersuchung nicht mehr gesagt werden, die m.E. überzeugend darlegt, daß das Anliegen des jungen Marx in der durchgeführten Kritik der politischen Ökonomie keineswegs verschüttet, sondern im Gegenteil von roman-

${ }^{1}$ E. Aveling, Charles Darwin und Karl Marx. Eine Parallele, in: Die Neue Zeit, 15/2, I 896/97, S. 745-757; L. Woltmann, Die Darwinsche Theorie und der Sozialismus. Ein Beitrag zur Naturgeschichte der menschlichen Gesellschaft, Düsseldorf r 899; J. Schaxel, Darwinismus und Marxismus. Ein Beitrag zur wissenschaftlichen Voraussetzung des Sozialismus, in: Der lebendige Marxismus. Festgabe zum 70. Geburtstage von Karl Kautsky, Jena 1924, S. 485-500; J. Schaxel, Marx, Engels und Darwin, in: Urania. Kulturpolitische Monatshefte über Natur und Gesellschaft, 5, 1928/29, S. 289-294; J. Boszotta, Einflüsse des Darwinismus auf den Marxismus, Diss. Wien I950; W. Rothmaler, Marx und Darwin, in: Wiss. Zeitschrift d. Universität Greifswald, math.-naturwiss. Reihe, 2, 1952/53, S. 3 г 3 ff.; O. Klohr, Marx und Engels über die Evolutionslehre Darwins, in: Wiss. Zeitschrift d. Martin-Luther-Universität Halle-Wittenberg, math.naturwiss. Reihe, 3, I953/54, S. 467 ff. Die beiden zuletzt genannten Aufsätze von Rothmaler und Klohr zeichnen sich gegenüber den übrigen Arbeiten dadurch aus, daß sie wenigstens annähernd alle erreichbaren Äußerungen von Marx und Engels heranziehen. Im übrigen aber sind sie wertlos. In der Darstellung geben sie lange Zitate, ohne auch nur den Versuch einer geistigen Durchdringung zu machen. Ich entdecke nur eine Ausnahme, und die ist ein Mißgriff: wenn Marx meint, Darwin entwickele seinen Gegenstand „grob englisch", deutet Rothmaler das auf die Übernahme von Malthus (S. 316). Die wirklichen Schwierigkeiten in der Interpretation der Texte werden gar nicht gesehen. In der Würdigung führen sie - in offensichtlicher Anlehnung an den "Darwin”-Artikel der 2. Auflage der Großen Sowjetenzyklopädie (Bd. I3, 1952, S. 367-370) und daher in bemerkenswerter Übereinstimmung - folgendes aus: Marx und Engels haben, im Gegensatz zu vielen Naturwissenschaftlern vom Fach, die epochemachende Bedeutung von Darwins Werk sofort erkannt. Sie verbanden dieses Urteil mit Kritik, und zwar mit sehr berechtigter Kritik, wie die Naturwissenschaft längst bewiesen hat. Aber zu ihrem Schaden ließ die Naturwissenschaft ihrerseits diese kritischen Hinweise lange Zeit unbeachtet. Erst die Sowjetbiologie zog sie aus der Vergessenheit wieder ans Licht und nicht zuletzt dem verdankt sie ihre großen Erfolge. - Vgl. auch: C. Huygens, Darwin - Marx. Bernstein als bestrijder van eene natuur-philosophische leer, Amsterdam 190I; A. Pannekoek, Marxismus und Darwinismus (Vortrag), Leipzig ı909; E. Mühlbach, Karl Marx über Darwins Lehre, in: Monistische Monatshefte, I4, 1929, S. 236-238; S. Hook, Marx and Darwinism, in: New Republic (New York), 67, 1931, S. 290. Nicht zugänglich waren mir die folgenden in den beiden Auflagen der Großen und in der Kleinen Sowjetenzyklopädie genannten Bücher: Darvinizm i marksizm, sbornik statej pod red. M. Ravič-Čerkasskogo, Char'kov 1925; B. Zavadovskij, Darvinizm i marksizm, Moskau-Leningrad 1926; I. Agol, Dialektičeskij metod i evoljucionnaja teorija, 3. Aufl. Moskau-Leningrad 193I; Učenie Darvina i marksizm-leninizm. Sbornik statej, pod red. P. Valeskalna, Moskau 1932; K. A. Timirjazev, C. Darvin i K. Marks (Sočinenija, t. 9), Moskau 1939. 
tischen Überspanntheiten befreit und positiv ausgeführt ist. Ein Rest ist jedoch geblieben: „noch der Aufklärung bedarf”, mit Jürgen Habermas zu sprechen, "die eigentümliche theoretische Nachgiebigkeit des ,ökonomischen' Marx, seit etwa 1858 , gegenüber dem ,metaphysischen' Engels ... der Einfluß der Engelsschen, Weltanschauung' auf Marx, der an einigen Stellen des ,Kapital' deutlich nachweisbar ist". 'Auf dieses Problem kann die Stellung, die Marx zu Darwin eingenommen hat, einiges Licht werfen.

In einem einleitenden Teil skizzieren wir Marx' und Engels' biographisch-persönliche Beziehung zu Darwin (I). Der Hauptteil der Arbeit über die sachliche Auseinandersetzung behandelt dann Marx und Engels getrennt, wobei wir weiter aufgliedern: in einem ersten Teil untersuchen wir jeweils Zustimmung und kritische Bemerkungen zu Darwin, die Interpretation des Darwinschen Werks, dessen verschiedene Aspekte ja durchaus unterschiedliche Deutungen durch die Zeitgenossen hervorriefen, und die bei dieser Interpretation auftretenden Mißverständnisse und Umdeutungen (II und IV); ein zweiter Teil schildert, wie Marx und Engels den Einfluß Darwins in ihren eigenen Auffassungen positiv verarbeitet haben (III und V). In einem Anhang klären wir die ungenauen Nachrichten der bisherigen Literatur über den Briefwechsel zwischen Marx und Darwin.

Engels hat Darwins Hauptwerk „On the Origin of Species” gleich nach dem Erscheinen 1859 gelesen, ${ }^{2}$ Marx erst ein Jahr später. ${ }^{3}$ Über weitere Darwin-Lektüre wissen wir bei Engels quellenmäßig nur noch, daß er 1868 das in diesem Jahr erschienene Buch „Variation of Animals and Plants under Domestication" studierte, ${ }^{4}$ das dürfte aber bei Engels' naturwissenschaftlichen Interessen nicht alles gewesen sein. Es ist möglich, daß Marx i 880 eine Veröffentlichung über Darwin plante, ${ }^{5}$ aber auch wenn das nicht der Fall gewesen sein sollte, kannte Marx sicherlich mehr als Darwins Hauptwerk; ${ }^{6}$ ob er wirklich alle Werke Darwins gelesen hat, wie Edward Aveling behauptet, wird

1 J. Habermas, Zur philosophischen Diskussion um Marx und den Marxismus, in: Philosophische Rundschau, 5, 1957, S. 177.

2 Engels an Marx, Dezember I 859, MEGA III/2, S. 447 f., als Tag wird der 11 . oder I 2. vermutet. Die I. Auflage der „Entstehung der Arten” erschien am 24. November 1859 und war sofort verkauft.

${ }^{3}$ Marx an Engels, 19. Dezember I860, ebd., S. 533.

- Engels an Marx, 22. Okt. r868, III/4, S. I16.

5 Darwin an Marx, 13. Okt. 1880; s.u. S. 465 f., 469.

"Man könnte z.B. vermuten, daß Marx zu seiner Notiz über die Korallen in „Kapital” I (S. $37^{8} \mathrm{Anm}$. 64) durch Darwins Buch iber die Korallenriffe angeregt worden ist. 
man vorsichtigerweise bezweifeln müssen, ${ }^{1}$ ausgeschlossen ist es bei den ungeheuren Büchermassen, die Marx bewältigt hat, natürlich nicht. Marx schätzte Darwin hoch. I873 sandte er ihm die zweite deutsche Auflage des „Kapital”. ${ }^{2}$ I 880 bat er ihn, die Widmung des zweiten Bandes des „Kapital" anzunehmen; Darwin lehnte ab, weil das eine gewisse "Zustimmung zum ganzen Werk implizieren” würde, das er jedoch nicht kenne, auch wolle er die religiösen Gefühle seiner Familie nicht verletzen - d.h. Darwin verband mit Sozialismus militanten Atheismus und wollte auch den leisesten Anschein vermeiden, diesen zu unterstützen. ${ }^{3}$ Auch Engels würdigte die eindrucksvolle Forscherpersönlichkeit Darwins, sein geduldiges Sammeln von Tatsachen und die Bescheidenheit seiner vielen Hinweise auf seine Vorgänger. ${ }^{4}$ Bei seiner Hochschätzung Darwins mußte Marx es um so tiefer bedauern, unter einer Sympathieerklärung für Rußland, die er heftig mißbilligte, auch Darwins Namen zu finden. ${ }^{5}$ Lebhaften Anteil nahmen Marx und Engels an Aufnahme und Ablehnung Darwins in der wissenschaftlichen Diskussion. ${ }^{6}$ Engels (und wohl auch Marx) war die Diskussion bekannt, die $1877 / 78$ in Deutschland nach einer Rede Virchows über die Beziehung zwischen Darwinismus und Sozialdemokratie geführt wurde. ${ }^{7}$ Anläßlich von Darwins Tod i 882

${ }^{1}$ E. Aveling, Charles Darwin und Karl Marx (s.o. S. 434, Anm. 1), S. 753, 756. Der elogenhafte Stil des Artikels legt den Verdacht von Úbertreibungen im Sachlichen nahe.

2 Darwin an Marx, I Okt. 1873, s.u. S. 464 f., $466 \mathrm{f}$.

${ }^{3}$ Darwin an Marx, I3. Okt. I 880 , s.u. S. 465 f., 467 f.

"Vorarbeiten zum "Anti-Dühring", Marx-Engels-Archiv, II, Frankfurt 1927, S. 405. Darwins Verhältnis zu seinen Vorgängern ist neuerdings von $C$. D. Darlington in ein kritischeres Licht gerückt worden (Darwin's Place in History, Oxford ig6o, S. 60-62).

${ }^{5}$ Marx an Engels, i i. Dez. 1876, III/4, S. 446.

${ }^{8}$ Engels an Marx, 8. April I863 (III/3, S. I 35), I6. Nov. I 864 (S. 203 f.); Marx an Engels, I8. Okt. I 868 (III/4, S. I I4); Engels an Marx, 18. März I869 (S. I69), 14. April i 869 (S. I 8 I f.); Marx an Engels, ro. Febr. 1870 (S. 274), 14. April I870 (S. 304); Engels an Marx, 21. Sept. 1874 (S. 425 f.), 28. Mai 1876 (S. 438); Marx an Engels, 4. Sept. I882 (S. 558). - Marx scheint, auch abgesehen von Fragen des Darwinismus, besonders auf Huxley geachtet zu haben (Briefe an Engels, 28. Jan. 1863 , IlI/3, S. 123; 12. Dez. 1866, S. $368 ; 9$. März I $870, \mathrm{III} / 4$, S. 290).

'Dialektik und Natur, in: Marx-Engels-Archiv, II, S. 215 , vermutlich auch S. IS4. Virchow attackierte in einer improvisierten Rede auf der so. Versammlung deutscher Naturforscher und Ärzte I 877 in München die vorschnelle Popularisierung des wissenschaftlich noch gar nicht einwandfrei gesicherten Darwinismus und wies dabei warnend auf die Wirkung hin, die eine solche Popularisierung bereits in den Köpfen ungebildeter sozialistischer Arbeiter anrichte: man nehme sie dort als wissenschaftliche Bestätigung des Sozialismus (Amtlicher Bericht, München 1877 , darin S. 68 f.). Die Rede wurde von Haeckel (Freie Wissenschaft und freie Lehre, Stuttgart 1878) und Oscar Schmidt (Darwinismus und Socialdemokratic. Vortrag, Bonn I 878) zurückgewiesen. Darwin stand der Verdächtigung, seine Lehre könne mit Sozialismus in Verbindung gebracht werden, verständnislos gegenüber („eine thörichte Idee”, Brief an Schetzer, 26. Dez, 1879, in: F. Darwin (Hg.), Leben und Briefe von Charles Darwin, übers. v. V. Carus, Stuttgart 
erwog Engels, einen Artikel über ihn zu schreiben, doch fand er neben der Arbeit an dem Aufsatz über „Bruno Bauer und das Urchristentum” nicht die Zeit, über einen konventionellen Gedenkartikel hinauszukommen, und so ließ er den Plan fallen. ${ }^{1}$ Es ist, wie bereits erwähnt, möglich, daß zwei Jahre zuvor auch Marx etwas über Darwin zu veröffentlichen beabsichtigte.

\section{II}

Als Darwin sein Hauptwerk veröffentlichte, hatte Marx seinen endgültigen geistigen Standort bereits seit langem bezogen, und Philosophen - Hegel und Feuerbach - waren es, die ihn dabei entscheidend bestimmt hatten. Mehr noch: im Ansatz des jungen Marx ist die Natur als solche ganz beiseitegesetzt; „die Natur, abstrakt genommen, für sich, in der Trennung vom Menschen fixiert, ist für den Menschen nichts", heißt es in "Nationalökonomie und Philosophie". ${ }^{2}$ Sieht man von den mathematischen Übungen ab, die Marx gleichsam zur geistigen Entspannung machte, so verbanden ihn später nur seine Studien zur ganz neuen Wissenschaft der Technologie, die er im Zusammenhang mit seinen nationalökonomischen Untersuchungen trieb, enger mit den Naturwissenschaften, wobei freilich sofort anzumerken ist, daß für Marx die technisch-ökonomische Aneignung der Natur im Zentrum seines Naturbegriffs steht, die Technologie also nicht als „angewandte” gegen die ,reinen” Naturwissenschaften herabgesetzt werden darf. ${ }^{3}$ Man muß sich das vergegenwärtigen, um Marx' Stellung zu Darwin in die richtigen Proportionen stellen zu können. Wie verschieden der Blickpunkt der beiden Männer war, wird vielleicht am deutlichsten, wenn man den folgenden Vergleich anstellt. Darwin entwickelte an Hand der Domestikation von Pflanzen und Tieren seine Theorie der natural selection, und er konnte dafür die reiche praktische Erfahrung von englischen Züchtern auswerten. Auch Marx kannte die Erfolge der englischen Tierzüchter, ${ }^{4}$ aber er sah im gezüchteten das „durch Arbeit veränderte” Tier. ${ }^{5}$ „Natur interessiert Marx in erster Linie als Moment menschlicher Praxis", und „die noch nicht gesellschaftlich vermittelte Natur” wird für ihn „,nur unter dem

I887, III, S. 229). - Marx notierte I 882 ironisch, daß Virchow sich Darwin überlegen fühle (an Engels, 4. Sept. I882, III/4, S. 558 ).

${ }^{1}$ Kautsky an Engels, II. Nov.; Engels an Kautsky, is. Nov. 1882, in: F. Engels, Briefwechsel mit Karl Kautsky, 2. Aufl. Wien 1955, S. 66, 69.

${ }^{2}$ K. Marx, Die Frühschriften, hg. v. S. Landshut, Stuttgart I953, S. 285.

${ }^{3}$ A. Schmidt, Der Begriff der Natur ... (s. o. S. 433, Anm. 3), passim.

- Das Kapital, II, Berlin 1958, S. 234.

${ }^{5}$ Das Kapital, I, Berlin 1959, S. I87. 
Aspekt möglicher Bearbeitbarkeit relevant", heißt es zusammenfassend bei Schmidt.1

Wir geben nun in chronologischer Reihenfolge die Äußerungen wieder, die Marx über Darwin gemacht hat, wobei wir zunächst das „Kapital” ausklammern.

In zwei Briefen, dem einen an Engels Ende 1860 , dem andren an Lassalle Anfang I86r, formulierte Marx sein erstes Urteil nach der Lektüre der „Entstehung der Arten”. Es läßt sich nach drei Gesichtspunkten gliedern.

x. „Das Buch” enthält, heißt es im Brief an Engels, „die naturhistorische Grundlage für unsere Ansicht";2 und präziser im Brief an Lassalle: „Darwins Schrift . . paßt mir als naturwissenschaftliche Unterlage des geschichtlichen Klassenkampfes". ${ }^{3}$ Es ist nicht mehr eindeutig bestimmbar, welche Seite des Darwinschen Buches Marx gemeint hat. Wenn er, was das wahrscheinlichste ist, Klassenkampf und Kampf ums Dasein parallelisieren wollte, so hat er dieses Urteil später nicht wiederholt.

2. Durch Darwin ist die Teleologie erledigt, zugleich aber „der rationelle Sinn derselben empirisch auseinandergelegt". ${ }^{4}$ D.h. Darwin zeigte, können wir in Marx' Sinne ergänzen, aus welcher Wurzel die Teleologie rührte, und erst damit leistete er wahrhaft wissenschaftliche Kritik. ${ }^{5}$ Nach Ruth-Eva Schulz urteilte Marx über die teleologische Geschichtsphilosophie, daß ihr der Schein zugrundeliege, daß die älteren Generationen der „Zweck” der jüngeren sind, ein Schein, der daraus entstehe, daß jede Generation von den Leistungen der Vergangenheit abhängig ist und sie in dieser Zuordnung auf sich be-

${ }_{1}$ A. Schmidt, a.a.O., S. 22, I 45 (Anm. 30). Ein schönes Beispiel über die Nachweise bei Schmidt hinaus ist das im Marx-Engels-Nachlaß im Internationalen Institut für Sozialgeschichte, Amsterdam, befindliche und unter der Nummer B. I 9 katalogisierte auf das Jahr I 876 datierte Oktavheft, das u. a. 5o Seiten Exzerpt aus M. J. Schleiden, Die Physiologie der Pflanzen und Thiere und Theorie der Pflanzencultur, Braunschweig 1850 , enthält (M. J. Schleiden / E. E. Schmid, Encyklopädie der gesammten theoretischen Naturwissenschaften in ihrer Anwendung auf die Landwirthschaft, Bd. 3). Das Exzerpt beginnt mit dem Abschnitt „Abhängigkeit der Culturpflanzen von äußeren Verhältnissen”, S. 357 ff., wobei außerdem noch Stellen hervorgehoben werden, die die landwirtschaftliche Produktion und ihre Technik, die Fruchtbarkeit des Bodens und die sie bedingenden Faktoren betreffen; als nächster Abschnitt folgt „Dic Ernährung der Culturpflanzen”, S. 33 I ff.; und dann erst hat Marx den Anfang des Werks, das mit der Pflanzenzelle beginnt, exzerpiert (S. 24 ff.).

${ }^{2}$ Marx an Engels, 19. Dez. 1860, MEgA III/2, S. 533.

3 Marx an Lassalle, I6. Jan. I86r, in: Der Briefwechsel zwischen Lassalle und Marx, hg. v. G. Mayer (Ferd. Lassalle, Nachgelassene Briefe und Schriften, Bd. 3), Stuttgart I9z2, S. 346 .

4 Marx an Lassalle, a.a.O.

- Vgl. die nicht genau parallele, aber doch sehr aufschlußreiche Stelle zur Religionskritik, Kapital I, S. 389 , Anm. 89 . 
trachtet. ${ }^{1}$ Nach Darwin ergibt sich etwas Ähnliches für die Natur: eine Kontinuität der Abänderung der Arten, die aber kraft des Selektionsmechanismus ganz atelisch verläuft und nur in der rückschauenden Betrachtung ,Richtung" hat. ${ }^{2}$

3. Marx bemängelte an Darwins Buch „die grob englische Manier der Entwicklung"3 (ein Jahr zuvor hatte Engels geschrieben, „die plumpe englische Methode" müsse man eben ,in den Kauf nehmen"4). Das ist an sich nichts Ungewöhnliches. Marx tadelte häufig an englischen Schriftstellern ihren Mangel an philosophischer Schulung, vor allem ihren abstraktionsarmen Empirismus. ${ }^{5}$ Es scheint jedoch, daß er die umständliche Gründlichkeit Darwins zu wenig würdigte und auch nicht berücksichtigte, daß die ungeheure Fülle des gesammelten Materials - Darwin schätzte sich selbst als einen Kompilator ein ${ }^{6}$ Darstellung und Entwicklung unförmig machen mußte.

I 862 griff Marx nach erneuter Lektüre der "Entstehung der Arten" in einem Brief an Engels die Behauptung Darwins auf, der Kampf ums Dasein resultiere aus der geometrischen Progression der Vermehrung, und das sei die These von Malthus' „Essay on Population”, übertragen auf Tiere und Pflanzen. ${ }^{7}$ Marx bemerkte dazu, daß dieser Satz nicht allzu wörtlich genommen werden dürfe. Bei Malthus liege ja gerade "der Witz" darin, daß die geometrische Progression des Menschen nicht für Tiere und Pflanzen gelte, sondern deren Ver-

${ }^{1}$ R.-E. Schulz, Geschichte und teleologisches System bei Karl Marx, in: Wesen und Wirklichkeit des Menschen. Festschrift für H. Plessner, Göttingen I 957, S. $153 \mathrm{ff}$.

${ }^{2}$ Anders interpretiert die I. Auflage der Bol'šaja Sovetskaja Enciklopedija in ihrem Artikel „Darvin i darvinizm” (Bd. 20, Moskau 1930, Sp. 430-471) die Briefstelle: nach Darwin ergebe sich "Zweckmäßigkeit” der Organismen aus dem Selektionsmechanismus als funktionelle Einpassung in den umfassenden Lebensraum der Umwelt (Sp. 446). Ebenso A. Schmidt, a.a.O., S. 83; die von ihm herangezogenen Stellen gehören jedoch in einen anderen Zusammenhang.

s Marx an Lassalle, a.a.O.; "grob englisch entwickelt" heißt es im Brief an Engels, a.a.O.

4 Engels an Marx, I 2. (?) Dez. 1859, III/2, S. 448.

'So z.B. bei Adam Smith, Kapital, II, S. I 85, 193. „Die Engländer” betrachten „getn die erste empirische Erscheinungsform einer Sache als ihren Grund", schrieb Marx generalisierend (Kapital, I, S. 423 Anm. I44).

- Darwin an Hooker, 24. Febr. 1863, in: Leben und Briefe (s.o. S. 436, Anm. 7), III, S. 8.

${ }^{7}$ Ch. R. Darwin, Über die Entstehung der Arten durch natürliche Zuchtwahl oder die Erhaltung der begünstigten Rassen im Kampfe um's Dasein, übers. v. V. Carus, 5. Aufl, Stuttgart 1872 , S. I6. Darwin war nach eigener Auskunft bei zufälliger Lektüre der Malthusschen Schrift im Oktober 1838 auf die Theorie der natürlichen Zuchtwahl gekommen (The Autobiography of Charles Darwin I 809-1 882. With original omissions restored, ed. by Nora Barlow, London 1958, S. 120; vgl. Darwin an Haeckel, 8. Okt, 1864, in: E. Haeckel, Natürliche Schöpfungsgeschichte, 9. Aufl. Berlin 1898, I, S. I 20). Neuerdings hat Darlington (s.o. S. 436, Anm. 4), S. 34 f., diese Auskunft angefochten und auf ihr rechtes $\mathrm{MaB}$ zurückgeführt. Darwin schätzte übrigens Malthus allgemein als „großen Philosophen" (Leben und Briefe, II, S. 308 ; vgl. III, S. 45), eine aufschlußreiche Tatsache für den Vergleich zwischen Darwin und Marx (zu Marx' Urteil über Malthus s.u. S. 442). 
mehrungsquote gegenübergestellt werde. ${ }^{1}$ Wenn Darwin, heißt es in den „Theorien über den Mehrwert”, geometrische Progression auch für 'Tiere und Pflanzen annehme, stoße er Malthus' Theorie geradezu um. Aber abgesehen davon finde sich bei Darwin auch im einzelnen, beispielsweise bei seinen Ausführungen ,über das Erlöschen von Arten ... die naturhistorische Widerlegung der Malthusschen Theorie". ${ }^{2}$ Diese Bemerkungen dürften nicht zutreffen. Die Wiedergabe von Malthus ist unrichtig ; ${ }^{3}$ und das, was Darwin zum Erlöschen von Arten sagt, bedeutet keine Einschränkung des allgemeinen Satzes, daß jede Art sich über ihre gegenwärtige Zahl hinaus zu vermehren strebt, sondern eine Aussage über die Stärke der dieser Vermehrung entgegenwirkenden Faktoren. ${ }^{4}$ Doch scheint soviel richtig zu sein, daß Darwin keine exakte Parallele zwischen der Vermehrung des Menschen und der der Tiere und Pflanzen ziehen wollte; er bemerkt übrigens in Übereinstimmung mit Malthus ${ }^{5}$-, der Druck der Übervölkerung laste auf Pflanzen und Tieren in verstärktem Maße, da diese ihre Nahrungsmittel nicht künstlich vermehren und sich auch nicht mit Überlegung von Befruchtung und Paarung enthalten könnten. ${ }^{6}$ - Völlig zutreffend und überdies viel wichtiger ist dagegen die Bemerkung, mit der Marx in seinem Brief an Engels fortfährt, Darwins Naturmodell erinnere sehr merkwürdig an bestimmte Aspekte der bürgerlichen Gesellschaft: „Teilung der Arbeit, Konkurrenz, Aufschluß neuer Märkte, ,Erfindungen' und Malthusscher ,Kampf ums Dasein"'.7 Darwin gewann seine Theorie aus der Erfahrung der gesellschaftlichen Wirklichkeit seiner Zeit, in der auch Malthus' Bevölke-

\footnotetext{
${ }^{1}$ Marx an Engels, 18. Juni $1862,111 / 3$, S. 77. tiert abdruckt.

4 Entst. d. Arten, S. 394 ff., bes. S. 396, 399.

${ }^{5}$ Bevölkerungsgesetz, I, S. Is, 23.

- Entst. d. Arten, S. 76.

${ }^{7}$ Marx an Engels, a.a.O., S. 78.
}

${ }^{2}$ Theorien über den Mehrwert, 2. Teil, Berlin 1959, S. I 10.

${ }^{3}$ Vgl. die wenigen Hinweise auf Pflanzen und Tiere bei Malthus, Eine Abhandlung über das Bevölkerungsgesetz usw., dt. Übers. nach d. 6. Aufl. I826, Jena 1905, I, S. I 4 f. An der in Anm. 2 genannten Stelle behauptet Marx, Malthus stelle eine arithmetische Progression der Vermehrung für Pflanzen und Tiere auf und stelle sie der geometrischen Progression der Menschen gegenüber. Es scheint, daß ihm eine Verwechslung mit der arithmetischen Progression der Vermehrung der Lebensmittel (Malthus, a.a.O., I, S. 2I) unterlaufen ist. Sollte Marx jedoch unter „Lebensmitteln” eben Pflanzen und Tiere verstanden haben - das ist die einzig mögliche Erklärung dieser Stelle -, so hätte et Malthus falsch verstanden; dieser meint Verbesserung der landwirtschaftlichen Nahrungsmittelproduktion. Nicht weiter helfen F. Krahl (Karl Marx über Th. R. Malthus, in: Dt. Zschr. f. Philos. 2, 1954, S. 384-417), der diese wichtige, aber eben schwierige Stelle einfach übergeht, und R. L. Meek (Marx und Engels über Malthus. Werk- und Briefauszüge gegen die Theorien von Thomas Robert Malthus. Hg. u. eingeleitet v. R. L. Meek, Berlin (Ost) I956), der sie in seiner Einleitung S. Io f. übergeht und auf S. I 4 o f. dann unkommen- 
rungstheorie schon materielle Gestalt annahm; soweit Darwin jedoch unmittelbar geistig von Malthus abhängig ist, ist zu sagen, daß Malthus' Theorie ihrerseits auf dem Hintergrund der steigenden Geburtenziffern und des Anwachsens der industriellen Reservearmee gesehen werden muß, die die Folge der englischen Armengesetzgebung von 1795 waren. ${ }^{1}$

Vier Jahre später, I866, äußerte Marx kritische Einwände gegen die „Entstehung der Arten”. Einmal lasse das Buch noch eine Reihe von Fragen ungelöst, z.B. die Entartung, zum andern ständen bestimmte Tatsachen Darwins Theorie im Wege, z.B. die Lücken in der Paläontologie (Darwins Erklärung der „Unvollständigkeit der geologischen Urkunden"2 befriedigte ihn also nicht) und die Wirkungen der bis zu einem gewissen Grad der Auseinanderentwicklung möglichen Bastardisierung zweier Varietäten. Vor allem aber hatte Marx auszusetzen, daß „der Fortschritt . . . bei Darwin rein zufällig” sei und daß man aus dem Buch, in der geschichtlichen und politischen Anwendung" nicht viel herausholen könne. ${ }^{3}$

I 868 ist dieses Urteil revidiert. Wenn Tiere und Pflanzen nach Darwin die natürliche Zuchtwahl bewußtlos und als einen außerhalb ihrer selbst sich vollziehenden Mechanismus erfahren, so erinnerte Marx das nun im Extrem an die bürgerliche Gesellschaft, der er bekanntlich vorwarf, daß sie ihre eigenen Bewegungsgesetze nicht durchschaue, geschweige denn beherrsche. Diese neue Stellungnahme gipfelt in dem Satz, Darwins Buch habe, wenn auch "unbewußt”, eine „sozialistische Tendenz".4 Mit dieser Aussage werden wir uns noch zu beschäftigen haben.

${ }^{1}$ E. Gruner, Vom Standort und den Aufgaben der Sozialgeschichte, in: Vjschr. f. Sozialu. Wirtschaftsgeschichte, 50, 1963, S. I55; vgl. A. Sauvy, Malthus et les deux Marx. Le problème de la faim et de la guerre dans le monde, Paris 1963, S. 55-58 und A. Schmidt, a.a.O., S. 37 .

2X. Kapitel, S. 356 ff.; die Erklärungshypothese S. 370, 379. Darwin sah selbst offene Fragen (S. 385 f.).

${ }^{3}$ Marx an Engels, 7. Aug. 1866, III/3, S. 355. Die Stelle ist eine Besprechung des Buches von P. Trémaux, Origine et transformations de l'homme et des autres bêtes, Paris 1865 , das Marx beim ersten Eindruck als "ein sehr bedeutender Fortschritt uber Darwin” erschien (a.a.O.). Auf Grund dieses Urteils konnten einige der oben genannten Punkte der Kritik negativ erschlossen werden. Der Brief ist für uns sehr wertvoll, nicht wegen der Zustimmung zu Trémaux (die Engels später so gut wie völlig zu widerlegen wußte, s. die Briefe an Marx vom 2. und s. Okt. und den Brief von Marx an Engels vom 3. Okt., III/3, S. $360 \mathrm{ff}$.), sondern wegen der Kritik an Darwin.

4 Wir können dies indirekt aus dem Urteil über das Buch von K. Fraas, Klima und Pflanzenwelt in der Zeit, ein Beitrag zur Geschichte beider, Landshut I 847, erkennen. Marx entdeckt erstens, daß F. schon vor Darwin eine Entstehung der Arten in der Zeit annimmt, und zweitens, daß er die anfänglichen Ergebnisse der Agrikultur zwar für nützlich, aber ihre langfristige Wirkung für schädlich hält, da sie den Boden seiner Feuchtigkeit beraube und schließlich zu Steppenbildung führe. Marx zieht daraus den Schluß, daß auch die 
I 870 ging Marx die 2. Auflage des Buches von Friedrich Albert Lange „Die Arbeiterfrage” zu. ${ }^{1}$ Lange stellt darin die These auf, der aus dem Druck der Übervölkerung resultierende Kampf ums Dasein sei ein allgemeines Gesetz, das gleichermaßen für die organische Natur wie für die menschliche Geschichte gelte. Auch die moderne Industriegesellschaft mache dabei keine Ausnahme, wofür sich Lange ausgerechnet auf Marx' „Kapital” beruft. ${ }^{2}$ Hier begegnete Marx also der Sozialdarwinismus in der Gestalt des Neomalthusianismus, d.h. als Wiederbelebung der Malthusschen Thesen, die durch den durchschlagenden Erfolg der Darwinschen Nachweise verstärkte Autorität gewannen. Wir müssen hier skizzieren, was Marx von Malthus' ,Essay on Population" hielt. Er sei, meinte er, in der I. Auflage ein einziges Plagiat, ${ }^{3}$ das die englische Oligarchie im Kampf gegen die Fortschrittsideen der französischen Revolution, also aus reinen „Parteiinteressen" hochgespielt habe; ${ }^{4}$ Malthus habe dann in den nächsten Ausgaben "oberflächlich kompiliertes Material in das alte Schema" gestopft. ${ }^{5}$ Seinerseits war Marx der Meinung, daß in der menschlichen Geschichte jede Produktionsweise ihr eigenes Bevölkerungsgesetz habe. ${ }^{6}$ In der gegenwärtigen kapitalistischen Produktionsweise wirke der Mechanismus, daß mit dem technischen Fortschritt in der $\mathrm{Zu}-$ sammensetzung des Kapitals der konstante Teil auf Kosten des variablen zunehme, die Nachfrage nach Arbeitskräften also tendenziell falle. So produziere die Arbeiterklasse „mit der durch sie selbst produzierten Akkumulation des Kapitals ... in wachsendem Umfang die Mittel ihrer eignen relativen Überzähligmachung" - das sei das Bevölkerungsgesetz der kapitalistischen Gesellschaft.? Lange meinte dazu, dieser Mechanismus sei keineswegs so speziell, daß et nicht

Agrikultur „bewußt beherrscht" getrieben werden müsse, und setzt hinzu, das Buch von F. habe demnach (!) „auch" (das bezieht sich auf Darwin) eine „sozialistische Tendenz unbewußt" (an Engels, 25. März 1868, III/4, S. 34).

${ }^{1}$ F. A. Lange, Die Arbeiterfrage. Ihre Bedeutung für Gegenwart und Zukunft, 2. Auf. Winterthur 1870 .

:S. I ff., I I $\mathbb{f}$.

3 Brief an Schweitzer, 24. Jan. 1865 , in: K. Marx/F. Engels, Ausgewählte Briefe, Stuttgart 1953, S. 182; Kapital, I, S. 369 , Anm. 51; S. 532, Anm. 325; S. 648, Anm. 75; Theorien über den Mehrwert, 2. Teil, S. 109. Überhaupt sprach Marx Malthus wissenschaftliche Qualifikation ab: et kenne nicht die rücksichtslose Wissenschaftlichkeit, die nur der eigenen Einsicht verpflichtet sei und durch die sich z.B. Ricardo ausgezeichnet habe, vielmehr sei er ein „Sykophant” der englischen Grundaristokratie, der deren Interessen (und, wenn damit übereinstimmend, auch die Interessen der Bourgeoisie) wider bessere Einsicht verfechte (Theorien über den Mehrwert, 2. Teil, S. 104 f.).

Kapital, I, S. 648, Anm. 75.

$s$ Ebenda.

S. 666.

7. 662-666, wörtliches Zitat S. 665. 
mehr unter das allgemeine Gesetz vom Kampf ums Dasein subsumiert werden könne, sondern nur eine leichte Variation desselben. Er erklärte die Anfänge des Fabrikwesens, die Frauen- und Kinderarbeit aus der Pauperisierung; die ,immer weiter treibende Noth" aber sei eben nichts „Andres, als eine Metamorphose des Kampfs um das Dasein". ${ }^{1}$ In einem Brief an Kugelmann schob Marx diese Ausführungen geradezu verächtlich beiseite. Man müsse untersuchen, schrieb er, wie sich der Kampf ums Dasein geschichtlich jeweils ausgeprägt habe; subsumiere man einfach die ganze Geschichte unter den Darwinschen Ausdruck „Kampf ums Dasein”, so zeuge das von „Denkfaulheit" und würdige den Ausdruck zur „Phrase" herab. " Damit bestritt Marx dem Sozialdarwinismus das Recht, Darwin für sich in Anspruch zu nehmen.

\section{III}

Was hatte Marx dem Sozialdarwinismus nun entgegenzusetzen? Eine Antwort darauf gibt das „Kapital”, das wir bisher ausgeklammert haben.

Es ist bekannt, daß Marx an zwei Stellen des „Kapital” ausdrücklich auf Darwin Bezug nimmt. Darwin weise, heißt es an der einen, auf „die Geschichte der natürlichen Technologie” hin, denn die Bildung und Veränderung von Organen bei Pflanzen und Tieren sei nichts anderes als die Herausarbeitung von deren „Produktionsinstrumenten". Marx bedauert, daß sich bisher noch kein Darwin für die Geschichte der menschlichen Technologie gefunden habe. ${ }^{3}$ An der anderen Stelle beruft sich Marx auf Darwins Entdeckung, daß ein Organ um so weniger variiert, je spezieller seine Funktion ist, und bezieht das auf die menschlichen Werkzeuge, bei denen sich Spezialität von Funktion und Form ebenso entsprechen. ${ }^{4}$ Hyppolite hat nun darauf aufmerksam gemacht, daß die Bezüge des „Kapital” auf Darwin sich darin nicht erschöpfen. ${ }^{5}$ Was er selbst dazu expliziert, ist jedoch ungenügend und nur als Hinweis darauf brauchbar, daß es sich um die historischen Teile handelt, deren Gegenstand die Genese des Kapitalismus ist.

Man weiß, daß Marx im Februar I 866 das Manuskript des „Kapital”

1 Lange, a.a.O., S. 202 ff., 213 ff., 223, wörtliches Zitat S. 223.

${ }^{2}$ Brief an Kugelmann, 27. Juni I 870, Ausgewählte Briefe, S. 275 f.

Sapital, I, S. 389, Anm. 89.

"S. 358, Anm. 31, wörtliches Zitat aus „Die Entstehung der Arten”, S. 169.

5 J. Hyppolite, De la structure du "Capital" et de quelques présuppositions philosophiques de l'œuvre de Marx, in: Études sur Marx et Hegel, Paris r955, S. 146; vgl. auch S. Hook (s.o. S. 434, Anm. I) und J. Boszotta (s.o. S. 434, Anm. I), S. Io5, I 37. 
erweiterte, und zwar angefangen vom historischen Teil des 8. Kapitels über den Arbeitstag ${ }^{1}$ bis zum I 3 . Kapitel über „Maschinerie und große Industrie". ${ }^{2}$ Leider enthält der Marxsche Nachlaß im Internationalen Institut für Sozialgeschichte in Amsterdam für diesen Teil des „Kapital" keinen früheren Entwurf, so daß sich kein Aufschluß über die Art der Erweiterung gewinnen läßt. Andererseits hat Marx diese Partie später nicht mehr verändert, wie ein Vergleich der I. und 2. deutschen Auflage (1867, I872/73) und der von Marx durchgesehenen französischen Übersetzung (I 872-75) ${ }^{3}$ ergibt. Wir sind also ausschließlich auf den im Februar ז866 entstandenen Text angewiesen. Er ist teilweise eine Verarbeitung von Darwins Hauptwerk, wie das folgende Referat zeigen soll. ${ }^{4}$

Marx behandelt die Genese des Kapitalismus, die sich in den beiden großen Epochen der Manufaktur und der großen Industrie vollzieht. Die Manufaktur unterscheidet sich zunächst nur quantitativ vom zünftigen Handwerksbetrieb: „durch die größere Zahl der gleichzeitig von demselben Kapital beschäftigten Arbeiter". ${ }^{5}$ Die nun stattfindende Kooperation der Arbeiter, die eine Reihe von mechanischtechnischen Vorteilen hat, ${ }^{6}$ verändert jedoch zugleich das Wesen der Arbeit: die individuelle Arbeit wird zur gesellschaftlichen. ${ }^{7}$ Das Kapital andererseits erhält die „Funktion der Leitung, Überwachung und Vermittlung" der einzelnen 'Tätigkeiten. ${ }^{8}$ Die Arbeitsteilung der Manufaktur hat einen doppelten Ursprung aus dem Handwerk. Einmal werden selbständige Handwerke so kombiniert, daß „sie nur noch einander ergänzende Teiloperationen im Produktionsprozeß einer und derselben Ware bilden". Zum andern wird ein einzelnes Handwerk ,in seine verschiednen besondren Operationen" zerlegt, bis ,jede derselben zur ausschließlichen Funktion eines besondren Arbeiters wird". Beidemal teilt die Manufaktur die Arbeit auf Grund des - um den Marxschen Lieblingsausdruck zu gebrauchen - „naturwüchsig” vorgefundenen Handwerks, sie hat daher nur eine „enge technische Basis”, die „wirklich wissenschaftliche Analyse des Pro-

1 S. 275 ff. Kapitelzählung nach der von allen späteren Ausgaben übernommenen 2. Auflage von 1872 .

${ }^{2}$ Marx an Engels, to. und 13. Febr. 1866, III/3, S. 305, 309.

${ }^{3}$ Das Kapital. Kritik der politischen Ökonomie. I. Buch: Der Produktionsprozeß des Kapitals, Hamburg 1867 ; 2. Auflage 1872 , das Nachwort ist datiert am 24. Januar 1873; Le Capital. Critique de l'économie politique. Traduction de M. J. Roy, entièrement revisée par l'auteur, Paris $1872-75$ (in Lieferungen).

"Vgl. zum Folgenden M. M. Rosental, Die Dialektik in Marx' „Kapital”, Berlin (Ost) I957, S. 131-136.

5 Kapital, I, S. 337.

S. 338-344.

'S. 345 .

${ }^{8}$ S. 346. 
duktionsprozesses" ausschließt. ${ }^{1}$ Der Arbeitsprozeß wird noch immer an den Arbeiter $\mathrm{m}$ t seinen Werkzeugen angepaßt. ${ }^{2}$ Die moderne große Industrie dagegen hat mit dem „Prinzip, jeden Produktionsprozeß, an und für sich und zunächst ohne alle Rücksicht auf die menschliche Hand, in seine konstituierenden Elemente aufzulösen", die Wissenschaft der Technologie hervorgebracht und den Produktionsprozeß verwandelt in ,bewußt planmäßige und je nach dem bezweckten Nutzeffekt systematisch besonderte Anwendungen der Naturwissenschaft". ${ }^{3}$ Mit anderen Worten: mit der maschinellen Industrie hat sich der Mensch endgültig von einer „natürlichen Technologie” entfernt, wie sie Marx bei Darwin angedeutet fand. Das zeigt sich auch und gerade an dem Phänomen, das die pervertiert-kapitalistische Form dieses Schrittes bezeichnet, daß die Maschinerie den Arbeiter in einen Teil ihrer selbst verwandelt, ihm gegenübertritt ,als tote Arbeit, welche die lebendige Arbeitskraft beherrscht und aussaugt", ${ }^{4}$ das ursprüngliche Verhältnis von Arbeitsmittel und Arbeiter also auf den Kopf stellt.

Dieser Umwälzungsprozeß hat tiefgreifende Wirkungen auf die physische und geistige Konstitution des Menschen. In der Manufaktur wird der Arbeiter auf eine bestimmte Detailfunktion spezialisiert, die er - das hängt mit der konservativen technischen Grundlage derManufaktur zusammen - meist lebenslang ausführt und deren „technische Kunstgriffe" sich daher auch von einer Arbeitergeneration auf die andere vererben. ${ }^{5}$ Darin läßt sich die Manufaktur noch mit den früheren Kasten und Zünften vergleichen, die auch schon "Teilarbeit in den Lebensberuf eines Menschen" verwandelt haben. ${ }^{6}$ Der Prozeß der Spezialisierung der menschlichen Tätigkeit entspricht überhaupt - hier spielt Marx deutlich auf Darwin an - dem Prozeß der Differenzierung der Arten in der Natur; jedoch im Unterschied zur Natur, in der das Individuum auf den Gesamtmechanismus gar keinen Einfluß hat, kann beim Menschen der Differenzierungsprozeß auf einer bestimmten Stufe durch gesellschaftliches Dekret, eben durch Kasten und Zünfte, fixiert werden. ${ }^{7}$ Jedoch bringt die Manufaktur etwas wesentlich Neues: sie treibt die vorgefundene „naturwüchsige Sonderung der Gewerbe . . . systematisch zum Extrem";8 die Detailfunktion deformiert und verkrüppelt den Körper des Arbeiters ${ }^{9}$ und vernichtet „die Spann- und Schwungkraft der Lebensgeister"10 in einem Maße, daß erstmals das Phänomen der ,industriellen Pathologie” auftaucht. ${ }^{11}$

Die Maschine jedoch, die, indem sie „Muskelkraft entbehrlich”

$\begin{array}{lllll}{ }^{1} \text { S. } 354 . & { }^{2} \text { S. } 397,404 . & { }^{3} \text { S. } 511 \text { f. } & { }^{4} \text { S. } 444 . & { }^{5} \text { S. } 355 . \\ { }^{\circ} \text { Ebenda. } & { }^{7} \text { S. } 356 . & { }^{8} \text { S. } 359 . & { }^{9} \text { S. } 366 \text { u. Anm. } 46,378 . \\ { }^{10} \text { S. } 357 . & { }^{11} \text { S. } 381 . & & & \end{array}$


machte, Frauen und Kinder in den Arbeitsprozeß schleuderte, ${ }^{1}$ hat auch alle Virtuosität der Manufakturarbeiter an sich gezogen und läßt, an die Stelle der künstlich erzeugten Unterschiede der Teilarbeiter ... vorwiegend die natürlichen Unterschiede des Alters und Geschlechts" treten. ${ }^{2}$ Das ist jedoch keine Rückkehr zur Natur, sondern bezeichnet nur einen quasi-natürlichen, genauer: unternatürlichen Zustand des Menschen, denn gerade die Natur kennt ja, wie Marx aus Darwin entnimmt, Differenzierung der Organismen. Das zeigt sich auch darin, daß Maschinenarbeit bei Kindern, intellektuelle Verödung" bewirkt, die nichts zu tun hat mit ,jener naturwüchsigen Unwissenheit, welche den Geist in Brache legt ohne Verderb seiner Entwicklungsfähigkeit, seiner natürlichen Fruchtbarkeit selbst". ${ }^{3}$ Darüber hinaus sieht sich jeder Arbeiter „als ein winzig Nebending vor der Wissenschaft, den ungeheuren Naturkräften und der gesellschaftlichen Massenarbeit, die im Maschinensystem verkörpert sind", ${ }^{4}$ er steht vor der in der Manufaktur begonnenen, nun vollendeten „Scheidung der geistigen Potenzen des Produktionsprozesses von der Handarbeit".5 Aber zugleich enthält das kapitalistische System den Widerspruch dazu: die revolutionäre technische Basis der maschinellen Produktion und die damit zusammenhängende nicht abreißende Umschichtung von Arbeitermassen erzeugen „Wechsel der Arbeit, Fluß der Funktion, allseitige Beweglichkeit des Arbeiters". 6 So produziert und braucht die große Industrie notwendig „das total entwickelte Individuum, für welches verschiedne gesellschaftliche Funktionen einander ablösende Betätigungsweisen sind", ${ }^{7}$ und darin deutet sich ein wichtiges Element der künftigen Gesellschaftsform an, nämlich der allgemeine ,technologische Unterricht ... in den Arbeiterschulen". 8 Charakteristisches Merkmal der jetzigen Gesellschaftsform ist dagegen die Degeneration der Arbeiter. Es ist bekannt, welche Fülle von Beispielen Marx, vor allem aus den enthüllenden Reports der englischen Fabrikinspektoren, gehäuft hat über die Schädigung und Zerrüttung der Gesundheit der Arbeiter im frühkapitalistischen Wirtschafts- und Fabriksystem. ${ }^{9}$ Diesen Degenerationsprozeß sieht Marx „nur durch beständige Absorption naturwüchsiger Lebenselemente vom Lande verlangsamt”. Das Land gilt ihm als Restgebiet des „principle of natural selection, das nur die kräftigsten Individuen aufkommen läßt”, ein Restgebiet, das zusehends verschwindet. ${ }^{10}$ Nur ein Element der gegenwärtigen Gesellschaft, die anarchische Konkurrenz der Kapitalisten untereinander, erinnert noch an den Kampf ums Dasein in der Natur. ${ }^{11}$
1 S. 413 .
2 S. $44 \mathrm{I}$,
${ }^{5}$ S. 444 , vgl. S. 379 .
gl. S. 366 f., 400, 455 (Ure-Zitat).
'S. 5 I2. 7 S. 5I3. ${ }^{8}$ S. 514.
S S. $419 . \quad$ S. 445.
$444,447,48 \mathrm{I}-483,486-497$.
${ }^{10} \mathrm{~S} .28 \mathrm{r}$.
11 S. 374 .
'S. 417, 418 (Anm. 133), 
Man sieht: diese Partie des „Kapital” hat Marx im Gespräch mit Darwin geschrieben. ${ }^{1}$ Man kann ferner verstehen, daß Marx ein halbes Jahr später, wie schon erwähnt, der Seufzer entschlüpft ist, man könne aus Darwins Hauptwerk ,in der geschichtlichen und politischen Anwendung" nicht viel herausholen. Es ist jedoch auch ein ganz anderes Urteil möglich. Marx zeigt ja, wie weit sich der Mensch - unwiderruflich - über den Weg der Kasten, des zunftmäßigen Handwerks, der Manufaktur und der maschinellen Industrie von natürlichen Zuständen entfernt, ja, die natürlichen Grundlagen seiner Existenz zerstört hat und nun einer durch und durch rationalen Gestaltung seiner Lebensbedingungen zustreben muß. Und eben das scheint Marx in seinem oben zitierten Brief an Engels von I 868 zu meinen, in dem er schreibt, Darwins Buch habe eine unbewußte sozialistische Tendenz. Nahm Marx aber so Darwin für sich in Anspruch, mußte es eine Herausforderung für ihn sein, wenn die Sozialdarwinisten dasselbe taten. Vergleicht man einmal die Marxschen Darlegungen, das reiche Gewebe von Beziehungen zwischen Technik der Produktivkräfte, gesellschaftlichen Verhältnissen und physischer und geistiger Evolution des Menschen unter diesen Bedingungen etwa mit den Ausführungen Langes, wird sogleich klar, auf wessen Seite die Überlegenheit liegt. Es ist daher sehr wohl möglich, wenn auch leider auf Grund der Quellenlage nicht mehr zu entscheiden, daß Marx Darwin persönlich von dieser Überlegenheit überzeugen und zu einer Erklärung bewegen wollte. ${ }^{2}$

Aber auch ohne eine Entscheidung dieser Frage lassen sich einige Aufschlüsse über den späten Marx gewinnen. Im Vorwort zur I. Auflage des „Kapital” hat Marx als „Endzweck” des Buches bezeichnet, „das ökonomische Bewegungsgesetz der modernen Gesellschaft zu enthüllen”. Dieses „Naturgesetz” erlaube nicht, „naturgemäße Entwicklungsphasen” zu ,überspringen”, man könne nur ,die Geburtswehen abkürzen und mildern". ${ }^{3}$ Dieser metaphorische Bezug auf die

1 Zwei bisher noch nicht bemerkte Anspielungen auf Darwins Hauptwerk könnten auch folgende Stellen des „Kapital” sein: $x$. „... eine Biene beschämt durch den Bau ihrer Wachszellen manchen menschlichen Baumeister. Was aber von vornherein den schlechtesten Baumeister vor der besten Biene auszeichnet, ist, daß er die Zelle in seinem Kopf gebaut hat, bevor er sie in Wachs baut" ( 186 ) - eine Anspielung auf das 8. Kapitel der „Entstehung der Arten” über „Instinkt”, in dem Darwin die einfache Zelle der Hummel, die geometrisch-regelmäßige Zelle der Korbbiene und als Zwischenform die Zelle der mexikanischen Melipona domestica behandelt; 2. „Dieselbe Wichtigkeit, welche der Bau von Knochenreliquien für die Erkenntnis der Organisation untergegangner Tiergeschlechter, haben Reliquien von Arbeitsmitteln für die Beurteilung untergegangner ökonomischer Gesellschaftsformationen" ( 188 ). Die beiden Stellen liegen also nahe beieinander. Dazwischen steht jener Passus, in dem Marx das gezüchtete Tier das „durch Arbeit veränderte" nennt (s.o. S. 437).

2 S.u. S. 469 .

${ }^{3}$ Kapital, I, S. 7 f. 
Naturgeschichte ist nicht zufällig, auch nicht der folgende direkte Vergleich im „Kapital”: ,abstrakt strenge Grenzlinien scheiden ebensowenig die Epochen der Gesellschafts- wie die der Erdgeschichte". ${ }^{1}$ Wir konnten zeigen, daß hinter dieser bekannten ${ }^{2}$ Hinwendung des späten Marx zu einem gewissen Evolutionismus eine Auseinandersetzung mit Darwin steht. Marx’ Selbstrezension des „Kapital”, er habe für die Gesellschaft „denselben allmählichen Umwälzungsprozeß” gezeigt, "den Darwin naturgeschichtlich nachgewiesen" habe, ${ }^{3}$ ist also kein "jugement ironique", wie Maximilien Rubel meint, ${ }^{4}$ sondern durchaus ernstzunehmen. ${ }^{5}$

So gewinnt nun auch das bekannte Nachwort zur 2. Auflage des „Kapital” vom Januar I 873 Kontur. Marx zitiert hier eine russische Rezension der I. Auflage, ${ }^{6}$ in der es heißt, Marx betrachte "die gesellschaftliche Bewegung als einen naturgeschichtlichen Prozeß", dessen Gesetze „Wollen, Bewußtsein und Absichten" der Menschen bestimmten, nicht umgekehrt. Jede Epoche der gesellschaftlichen Entwicklung aber habe ebenso wie die Pflanzen- und Tierorganismen ihr spezifisches Strukturprinzip. Wenn es z.B. kein generelles, sondern nur ein für jede Epoche eigentümliches Bevölkerungsgesetz gebe, dürfe man die Gesetze der OOkonomie nicht länger mit denen der Physik und Chemie vergleichen, sondern müsse "das ökonomische Leben" mit „der Entwicklungsgeschichte auf andren Gebieten der Biologie” in Beziehung setzen. Marx stimmt dieser Wiedergabe vorbehaltlos zu und schließt an, eben das sei die dialektische Methode. ${ }^{7}$ Und etwas später folgen dann die berühmten Worte, er, Marx, habe die von Hegel herausgearbeiteten, allgemeinen Bewegungsformen" der Dialektik nicht wie Hegel auf die Idee, sondern auf den realgeschichtlichen Prozeß angewandt und „den rationellen Kern" der Hegelschen Dialektik aus „der mystischen Hülle" befreit. ${ }^{8}$ Wie Marx hier Methode und Grundgedanken seines Werks bestimmt, bereitet insofern Schwierigkeiten, als es nicht nur dem Verhältnis zu Hegel in den

\footnotetext{
S. $3^{88 .}$

${ }^{2}$ Fetscher, Von der Philosophie ... (s.o. S. 433, Anm. I), S. 44.

${ }^{3}$ Brief an Engels, 7. Dez. 1867, III/3, S. 460. Engels hat diese Selbstrezension fast wörtlich an die Stuttgarter Zeitung „Der Beobachter” eingesandt, wo sie am 27. Dez. I 867 erschien (abgedruckt in Marx-Engels-Archiv, II, S. 45 I-453). Den Vergleich mit Darwin unterstrich er noch: nannte Marx seine Methode materialistisch, setzte Engels "naturhistorisch" hinzu (452).

${ }^{4}$ M. Rubel, Karl Marx. Essai de biographie intellectuelle, Paris 1957, S. 436.

5 Vl. A. Schmidt, a.a.O., S. 34.

${ }^{6}$ Gemeint ist der Artikel des Petersburger Professors J. J. Kaufmann, Točka zrenija politiko-ekonomičeskoj kritiki u Karla Marksa, in: Vestnik Evropy, Mai 1872.

'Kapital, I, S. I6 f.

8 S. 18.
} 
Frühschriften widerspricht, ${ }^{1}$ sondern als, wie Schmidt glänzend gezeigt hat, „gerade auch das Denken des späteren Marx stärker von Hegelschen Positionen bestimmt ist, als Marx selbst” hier „Wort haben" will. ${ }^{2}$ Marx deutet hier eine Reduktion der Dialektik auf „Grundgesetze" an, die ähnlich denen, die Engels auf die Natur anwenden möchte, dem realgeschichtlichen Prozeß äußerlich gegenüberstehen, und diese Schwierigkeit wird auch von Schmidt nicht aufgeklärt. ${ }^{3}$ Das eigentliche Problem ist dabei, wie Marx den Ausführungen des Rezensenten, die einen strengen Soziologismus begründen, zustimmen konnte, denn aus dieser Zustimmung folgt alles weitere. Wir können jetzt angeben, was ihn dazu veranlaßt hat: Marx fand eben die Pointe getroffen, mit der er in den historischen Teilen des „Kapital” die gesellschaftliche Entwicklung zum Kapitalismus hin gezeichnet hatte, nämlich den angedeuteten Bezug auf die von Darwin entworfene Evolution der Natur. Es kam Marx hier nicht darauf an, daß der Rezensent das Bild insofern verkürzt hat, als Marx' Darstellung sich nicht in der einfachen Analogie erschöpft, daß die Arten in der Natur wie die Produktionsweisen in der Geschichte langsame Veränderungen und qualitative Unterschiede zeigen, sondern die Natur auch als Ausgangspunkt der gesellschaftlichen Entwicklung betrachtet, von dem her sich deren Richtung bestimmen läßt.

Wie Marx sich damit gewissen Positionen seines Freundes Engels näherte, wird sich noch ergeben. Hier sei nur bemerkt, daß Engels sich auf dieses Nachwort von 1873 berufen hat ${ }^{4}$ und daß ihm der historische Abschnitt des „Kapital”, den wir referierten, als ein Musterbeispiel für eins der Gesetze erschien, die er aus dem Bestand der Hegelschen Dialektik für sein weltanschauliches System übernehmen wollte, den Umschlag von Quantität in Qualität und umgekehrt. ${ }^{5}$ Dieser Annäherung von Marx an Engels war jedoch eine Grenze gesetzt: die Differenz in der Anthropologie. Wie Bollnow ausgeführt hat, bestimmt Engels das Wesen des Menschen nur negativ: unbe-

${ }^{1}$ S.o. das Zitat von Habermas, S. 435.

A. Schmidt, a.a.O., passim, Zitat S. I57, Anm. 43.

${ }^{3}$ A. Schmidt, a.a.O., S. 33-39, für Engels s. S. 43-46. Unbrauchbar sind die Ausführungen von Rubel, a.a.O., S. 325 .

- Ludwig Feuerbach und der Ausgang der klassischen deutschen Philosophie, Werke Bd. 21 , S. 293.

${ }^{5}$ Anti-Dühring, Werke Bd. 20, S. I 8 . Dabei dachte Engels wohl vor allem an die Stelle, an der Marx auseinandersetzt, daß eine bestimmte Geldsumme erforderlich ist, um einen mittelalterlichen Handwerksmeister in einen Kapitalisten zu verwandeln, und dabei auf Hegels Umschlag von Quantität in Qualität verweist (Kapital, I, S. 323); auf diese Stelle hatte Marx gleich beim Korrekturlesen im Brief vom 22. Juni 1867 Engels aufmerksam gemacht. 
schränkt, nicht-einseitig usw., der Weg des Menschen zu dieser Bestimmung ist also unendlich. „Für Marx dagegen ist die reale Verwirklichung des Gattungswesens der Menschen in sein Dasein im Laufe begrenzter Zeit deshalb möglich, weil dieses Wesen ex natura (materiell) begrenzt und bestimmt ist." $\mathrm{Daß}$ damit die Position nicht nur des jungen Marx beschrieben ist, zeigt eine Stelle in den „Theorien über den Mehrwert", an der Marx seine Geschichtsphilosophie in einer Auseinandersetzung mit Ricardo und Sismondi entwickelt. Ricardo betrachte, schreibt Marx, „die kapitalistische Produktionsweise als die vorteilhafteste für die Produktion überhaupt", und damit habe er "für seine Zeit" recht. Die Produktion als Selbstzweck, die Ricardo wolle, heiße nichts anderes ,als Entwicklung der menschlichen Produktivkräfte, also Entwicklung des Reichtums der menschlichen Natur als Selbstzweck”. Dem dürfe man nicht sentimental „das Wohl der einzelnen" gegenüberstellen, wie Sismondi das tue. Denn die „Entwicklung der Fähigkeiten der Gattung Mensch” habe sich in der bisherigen Geschichte nur durchgesetzt und durchsetzen können "auf Kosten der Mehrzahl der Menschenindividuen und gar Menschenklassen", und in der Natur sei es geradeso, daß die Vorteile der Gattung nur als Vorteile bevorzugter Individuen zur Geltung kämen. Schließlich aber werde beim Menschen dieser Antagonismus durchbrochen, und die Entwicklung aller Individuen falle mit der Entwicklung der Gattung zusammen. ${ }^{2}$

Diese Äußerung rundet zugleich das Bild, das wir von Marx' Verhältnis zu Darwin gezeichnet haben. Bei aller Entfernung des Menschen von den natürlichen Lebensbedingungen seit dem Beginn des Kapitalismus und ansatzweise schon früher, wie Marx sie im „Kapital” geschildert hat, scheidet der Mensch von einem bestimmten Aspekt aus gesehen, nämlich dem Verhältnis zwischen Individuum und Gattung, erst mit der klassenlosen Gesellschaft aus dem Reich der Natur.

\section{IV}

Wir wenden uns Engels zu. Dabei werden wir von vornherein erwarten können, daß nun die Quellen reichlicher fließen, waren Engels doch bei der wissenschaftlichen „Arbeitsteilung” mit Marx die Naturwissenschaften zugefallen. Insbesondere nach dem Rückzug aus dem Geschäftsleben ( 1870 ) warf er sich auf dieses Gebiet und ,machte”, um seine eigenen Worte zu gebrauchen, „eine vollständige mathematische und naturwissenschaftliche ,Mauserung' ... durch". ${ }^{3}$

1 Engels' Auffassung ... (s.o. S. 433, Anm. 2), S. $128 \mathrm{f}$.

2 Theorien über den Mehrwert, 2, Teil, S. I06 f.

3 Vorrede zur 2. Auflage des „Anti-Dühring" (x 885), Werke, Bd. 2o, S. Io f. 
Schon aus dem enthusiastischen Brief, den er unmittelbar nach der Lektüre der „Entstehung der Arten” an Marx schrieb, kann man erkennen, wohin seine Zustimmung zielte. Es heißt da:

„Die Teleologie war nach einer Seite hin noch nicht kaputt gemacht, das ist jetzt geschehn. Dazu ist bisher noch nie ein so großartiger Versuch gemacht worden, historische Entwicklung in der Natur nachzuweisen, und am wenigsten mit solchem Glück."1

Untersuchen wir die beiden Punkte, die Engels hier hetausstellt!

Unter Teleologie verstand Engels, wie er an anderer Stelle pointiert formulierte, die Auffassung, ,wonach die Katzen geschaffen wurden, um die Mäuse zu fressen, die Mäuse, um von den Katzen gefressen zu werden, und die ganze Natur, um die Weisheit des Schöpfers darzutun", ${ }^{2}$ er dachte also dabei, was festzuhalten ist, an die Physikotheologie. Was von den theologischen Bindungen, die die Naturwissenschaft nach Engels bis in die Mitte des I8. Jahrhunderts hatte, ${ }^{3}$ noch in säkularisierter Form übrig ist, ist mit Darwin endgültig dahin. So beobachtet Engels mit Befriedigung, wie die Verteidiger Darwins in England zum Angriff auf das Christentum übergehen. ${ }^{4}$ Werfen wir hier einen Blick auf Marx, so finden wir keine einzige derartige Stelle bei ihm.

Der zweite Punkt, die „Historisierung der Natur”, wie Engels” Biograph Gustav Mayer es formuliert hat, ${ }^{5}$ ist das zentrale Anliegen der „Naturdialektik”, Engels' (unvollendet gebliebenem) naturwissenschaftlichen Hauptwerk. Engels erkannte beim Studium der Geschichte der Naturwissenschaft, daß noch bis ins I 8. Jahrhundert die Natur als ein fixes System galt, dessen Elemente nach unveränderlichen Gesetzen aufeinander bezogen sind. ${ }^{6}$ Im Prozeß des Umsturzes dieses Weltbildes, dem Engels viel Aufmerksamkeit zuwandte, ${ }^{7}$ ragten für ihn drei Ereignisse heraus : die Entdeckung des Gesetzes der Umwandlung der Energie durch Mayer, Joule und Colding, die Entdeckung der Zelle durch Schwann und Schleiden und die wesentlich von Darwin durchgesetzte Evolutionstheorie. ${ }^{8}$

12. (?) Dez. 1859, חII/2, S. 447 f.

2 Dialektik und Natur (zitiert nach: Marx-Engels-Archiv. Zeitschrift des Marx-EngelsInstituts in Moskau, hg. v. D. Rjazanov, Bd. II, Frankfurt 1927, S. I I 7 f.), S. 243.

A.a.O.

4 Engels an Marx, 8. April i 863, III/3, S. 135.

5 Friedrich Engels. Eine Biographie, 2. Aufl., Den Haag 1934, Bd. II, S. 306.

- Dial. u. Natur, S. 242 f., vgl. S. 174 f.

7 S. $175,244 \mathrm{ff}$.

${ }^{8}$ S. 382 f.; Ludwig Feuerbach und der Ausgang der klassischen deutschen Philosophie, Werke, Bd. 21, Berlin 1962, S. 294 f. Die ersten beiden Entdeckungen würdigte Engels 
Nun war Engels überzeugt, daß die Naturwissenschaft zur Einordnung ihrer Ergebnisse der Philosophie nicht entraten könne ${ }^{1}$ und daß sie dieser Hilfe um so dringender bedürfe, je mehr sie in Abkehr vom alten Weltbild mit evolutionären Prozessen arbeite. Für solche Prozesse und ihre gleitenden Übergänge aber gebe nur die dialektische Philosophie, als deren Kronzeugen Engels in diesem Zusammenhang immer wieder Hegel anrief, ${ }^{2}$ die angemessenen Kategorien an die Hand. ${ }^{3}$ Es ist daher nicht überraschend, daß Engels die Evolutionstheorie gleichsam exemplarisch philosophisch zu interpretieren versuchte, besonders angesichts des philosophischen Unvermögens, das er - wie übrigens auch Marx - bei den darwinistischen Naturwissenschaftlern zu beobachten glaubte. ${ }^{4}$

Er unternahm diesen Versuch in drei Richtungen. Erstens in der Analyse des begrifflichen Ergebnisses: er schilderte, wie durch die Evolutionstheorie der starre Artbegriff gewissermaßen „flüssig” werde, sich in den nuancierten Übergängen sozusagen auflöse. ${ }^{5}$ In der Tat werden bei Darwin die Grenzen zwischen Species und Varietäten fließend. ${ }^{6}$ Zweitens zeichnete Engels den Forschungsgang nach: er wies darauf hin, daß früher die allgemeinen Bestimmungen, in unserem Zusammenhang die Merkmale einer Art, als notwendig und zugleich als der alleinige Gegenstand der Wissenschaft galten, während die zufälligen Attribute der Individuen unbeachtet blieben. Darwin dage-

im Brief an Marx vom 14 . Juli 1858 , also vor der Veröffentlichung der Darwinschen Theorie als die beiden epochemachenden Leistungen der modernen Naturwissenschaft (III/2, S. 326).

${ }^{1}$ Dial. u. Natur, S. 264; Engels setzt hinzu, die Naturwissenschaft könne sich zwar unphilosophisch gebärden, sie gerate aber in dieser Naivität gegenüber ihrem Angewiesensein auf die Philosophie dann meistens unter den Einfluß schlechter Modephilosophen.

2 Engels an Marx, 2 r. Sept. 1874, III/4, S. 425; Dial. u. Natur, S. 155, 187,201 f., 202 f., $234,258,272,285$.

${ }^{3}$ S. I52; zum Unterschied zwischen „Metaphysik" und „Dialektik” s. auch „Anti-Dühring", S. 20-23, I 12 f. und „Dialektik und Natur" passim.

"Marx an Engels, I2. Dez. I866, III/3, S. 368; I4. April I870, III/4, S. 304 . Auch der Schluß der berühmten Anmerkung 89 Kapital I, S. 389 („Die Mängel des abstrakt naturwissenschaftlichen Materialismus, der den geschichtlichen Prozeß ausschließt, ersieht man schon aus den abstrakten und ideologischen Vorstellungen seiner Wortführer, sobald sie sich über ihre Spezialität hinauswagen") scheint mir darwinistische Naturwissenschaftler zu meinen. Engels schrieb dem Mangel an philosophischer Besinnung zu, wenn einige englische Naturforscher wie A. R. Wallace, der Mitbegründer der Selektionstheorie, dem Spiritismus verfielen (Dial. u. Natur, S. 215). Er glaubte andererseits, wenigstens Ansätze einer „Rückkehr zu einer wirklich denkenden Naturanschauung” in England zu beobachten (Brief an Marx, 21. Sept. 1874, III $/ 4$, S. 425 f.).

${ }^{5}$ Dial. u. Natur, S. 189.

${ }^{6}$ Entstehung der Arten, S. 66, 491, 559, 564 f., vgl. S. 71 f. und Die Abstammung des Menschen und die geschlechtliche Zuchtwahl, übers. v. V. Carus, Stuttgart I871, Bd. I, S. $200 \mathrm{f}$. Varietäten können als ,beginnende Species” verstanden werden (Entstehung d. Arten, S. 72 u. 123 ). 
gen sei ausgegangen „von der breitesten vorgefundenen Grundlage der Zufälligkeit", von der unendlichen Skala der bekannt gewordenen Varietäten, und sei von da aus unausweichlich dahin geführt worden, den alten Artbegriff preiszugeben. ${ }^{1}$ Das ist so weit einleuchtend; problematisch ist jedoch die Schlußfolgerung, die Engels zieht: Darwin habe für die Hegelschen Sätze von der inneren Einheit von Notwendigkeit und Zufälligkeit, die die Naturwissenschaft als „paradoxe Spielereien" verachtet habe, ${ }^{2}$ "die praktische Beweisführung” geliefert. ${ }^{3}$ Darwin selbst verzichtet nämlich darauf, nach dem Zusammenbruch der alten Auffassung neu zu bestimmen, welche Merkmale eines Individuums oder einer Varietät „notwendig”, welche „zufällig” sind. Nun ist grundsätzlich nichts dagegen einzuwenden, wenn versucht wird, ein Untersuchungsergebnis unmittelbar zu vervollständigen und weiterzuführen; in diesem Fall aber läuft die Weiterführung, nämlich der Versuch einer neuen Unterscheidung von notwendigen und zufälligen Merkmalen, dem Ergebnis, Darwins Auflösung des Klassifizierungsschemas, diametral zuwider. Doch ist der Sinn der Engelsschen These nicht ganz eindeutig zu erfassen. Man könnte eventuell auch - und das wäre durchaus sinnvoll - so verstehen: nach Darwin kann keine noch so unscheinbare und geringfügige Varietät und damit letztlich auch kein Individuum einer Art untergeordnet und so ihr gegenüber für gleichgültig erklärt werden; jedes Individuum ist ebenso gleichrangig zufällig wie als solches „notwendig”. - Drittens versuchte Engels, Darwins Theorie als Theorie zu interpretieren. Er polemisierte gegen Haeckels Auffassung, die Evolutionstheorie sei induktiv erschlossen. ${ }^{4}$ Engels wies darauf hin, daß der Induktionsschluß nur problematisch ist. ${ }^{5}$ Eben dieses Urteil - induktiv, nur problematisch - fällte Darwin über seine Theorie $;^{6}$ Engels dagegen ließ sich von seiner eben erwähnten Folgerung leiten, Darwin sei zu einer neuen „Notwendigkeit” durchgestoßen. Er meinte ferner, die Induktion gelange nur zu Klassifikationen, und die seien ja gerade durch Darwin in Frage gestellt; die Evolutionstheorie deduziere im wörtlichsten Sinne, d.h. sie leite verschiedene Arten durch Abstammung

${ }^{1}$ Dial. u. Natur, S. 264 f., 267 , wörtliches Zitat S. 267.

2 S. 267 .

${ }^{3}$ S. 281 .

${ }^{4}$ Nach Haeckel wird dann nur auf einzelne noch nicht nachgewiesene Tatsachen deduktiv geschlossen: Anthropogenie oder Entwicklungsgeschichte des Menschen, 2. Aufl. Leipzig I 874, S. 82 f., 372; Natürliche Schöpfungsgeschichte, (1868) 9. Aufl. Berlin I 898, Bd. II, S. 796 f. Beispiel ist Goethes Entdeckung des Zwischenkiefers: Natürl. Schöpfungsgeschichte, Bd. I, S. $76 \mathrm{f}$.

5 Dial. u. Natur, S. 260.

${ }^{B}$ Entstehung d. Arten, S. 558 f., vgl. S. 455 . 
voneinander ab. ${ }^{1}$ Hier ist das, was mit dem Begriff der Evolution sachlich ausgesagt wird, und das methodologische Problem der Aufstellung einer Theorie der Evolution hoffnungslos miteinander vermengt. Die Ursache dafür dürfte in Engels' naiv-realistischer Erkenntnistheorie zu suchen sein.

Doch wie groß auch immer Engels' Mißverständnis der Evolutionstheorie war, gegen ihre eindeutige und nicht falsch zu verstehende Konsequenz, die Einbeziehung des Menschen in den Evolutionsprozeß, hatte er nichts einzuwenden. Im Gegenteil. Schon 1858 kam er beim Studium der vergleichenden Physiologie auf den Gedanken, der Unterschied zwischen dem Menschen und den Säugetieren sei kleiner als allgemein angenommen. ${ }^{2}$ Wir werden später sehen, wie Engels sich die Integrierung des Menschen in den Naturprozeß dachte.

Auch die Selektionstheorie Darwins hielt Engels für erläuterungsbedürftig. Er wies darauf hin, daß der „Kampf ums Dasein” nicht immer ,als direkte, körperliche Bekämpfung oder Verzehrung, sondern auch als Kampf um Raum und Licht" vorgestellt werden müsse. ${ }^{3}$ In der Tat wollte Darwin den Ausdruck, ,in einem weiten und metaphorischen Sinne" verstanden wissen. ${ }^{4}$ Ferner machte Engels auf das wichtige Moment aufmerksam, daß der Selektionsmechanismus auch ohne den Druck von Übervölkerung wirksam werden könne, dann nämlich, wenn sich die Lebensumstände verändern und die Angepaßtesten als solche überleben. Komme in einem solchen Fall Übervölkerung vor, so sei sie nur ein sekundäres Element und bewirke höchstens eine Beschleunigung des Abänderungsprozesses. Bei der sexuellen Zuchtwahl falle der Druck der Übervölkerung überhaupt weg. ${ }^{5}$ Den Vergleich zwischen der natürlichen Zuchtwahl und der Domestikation von Tieren und Pflanzen hielt Engels für schlagend; er bemerkte, um den Hintergrund der Darwinschen Entdeckung zu beleuchten, daß England das klassische Land der Züchter sei. ${ }^{6}$ Er scheint auch die eigentliche Pointe der Selektionstheorie, daß nämlich die Selektion nach dem statistischen Gesetz der großen Zahl sich durchsetzt, daß es daher nicht auf das einzelne Individuum, sondern auf lange Zeiträume und kleine Modifikationen ankommt, ${ }^{7}$ wenigstens geahnt zu haben, wenn er einen Unterschied zwischen dem Bestehen

1 Dial. u. Natur, S. 260.

${ }^{2}$ Brief an Marx, 14. Juli I 858, IIl/2, S. $326 \mathrm{f}$.

${ }^{3}$ Anti-Dühring, S. 63.

Entstehung d. Arten, S. 75, vgl. die Beispiele S. 75 f.

${ }^{5}$ Dial. u. Natur, S. 282 f.

${ }^{6}$ Anti-Dühring, S. 63; von P. Lafargue wiederholt (Der wirthschaftliche Materialismus nach den Anschauungen von Karl Marx [Socialdemokratische Bibliothek, 9], HottingenZürich I 886, S. 20).

? Entstehung d. Arten, S. I09, 115 , I 21. 
einer Art und eines Individuums macht: das Nebeneinander von bestehenden Arten möge notwendig sein, für das Individuum bleibe es zufällig.' Schließlich erkannte Engels, daß Darwin in der Regel von den (damals auch noch weitgehend unbekannten) Ursachen der Modifikationen absieht und vor allem verfolgt, wie sich die Modifikationen unter den Bedingungen der Selektion „festsetzen". ${ }^{2}$

Bei aller Zustimmung zur Evolutionslehre nun betrachtete Engels die Selektionstheorie Darwins als vorläufig. ${ }^{3}$ In der Natur gebe es nicht nur Kampf, sondern auch „Zusammenwirken”; man müsse beides zusammennehmen, sonst werde man einseitig. ${ }^{4}$ Von diesem Vorwurf sei Darwin nicht ganz freizusprechen, er habe, typisch für einen Entdecker, seiner Theorie „einen übermäßigen Wirkungskreis" zugeschrieben. ${ }^{5}$ Engels glaubte, durch Haeckels Theorie von Anpassung und Vererbung als den zwei Prinzipien der Evolution ${ }^{6}$ die Annahme vom Kampf ums Dasein auf ihr rechtes Maß zurückführen zu können. ${ }^{7}$ Doch dieser Versuch ist bereits ein Reflex der Polemik gegen den Sozialdarwinismus.

Auch Engels begegnete der Sozialdarwinismus in der Gestalt des Neomalthusianismus. So versuchte er, Darwin möglichst von Malthus zu trennen. Zunächst machte er einen methodischen Vorbehalt: sicherlich gehe in der Natur ein Ausgleich des Mißverhältnisses zwischen der großen Zahl der Keime und der kleinen Zahl der zur vollen Ausbildung gelangenden Organismen vor sich, doch seien die spezifischen Bevölkerungsgesetze, die die organische Natur ebenso wie der Mensch habe, noch fast ganz unerforscht, und ohne derartige Untersuchungen könne man über Einzelheiten dieses Ausgleichsprozesses nichts aussagen. Darwins Aussage, die wir bereits analysierten, er habe die Malthusschen Thesen auf die Natur übertragen, hielt Engels entgegen, man brauche „keine Malthussche Brille”, um den Kampf ums Dasein in der Natur wahrzunehmen. ${ }^{8}$ Es ist richtig, wenn

1 Dial. u. Natur, S. 266. Die Stelle findet sich zwar unter den Bemerkungen zum Determinismus, scheint mir aber das erwähnte Problem zu berühren. Allerdings trifft sie es nicht genau; Engels sieht es vom Individuum aus („es ist für [!] das einzelne Tier zufällig”), und das ist überhaupt, wie wir weiter unten an Hand seiner Außerungen über die Anpassungsfäbigkeit des organischen Lebens zeigen werden, für ihn typisch.

${ }^{2}$ Anti-Dühring, S. 65 ; vgl. Entst. d. Arten, S. 86 f., 124 f., 148.

${ }^{3}$ Brief an Lavrov, I 2.-1 7. Nov. 1875, in: K. Marx/F. Engels, Ausgewählte Briefe, Stuttgart 1953, S. 356. Diese Stellung zu Darwin wurde dann für die Sozialisten typisch, s. z.B. Aveling, Charles Darwin und Karl Marx (s.o. S. 434, Anm. 1), S. 75 I.

- Brief an Lavrov, a.a.O.

${ }^{5}$ Anti-Dühring, S. 65.

- Haeckel, Natürl. Schöpfungsgeschichte, I, S. 137 ff., bes. S. I47.

"Dial. u. Natur, S. 283, vgl. auch die Zustimmung zu Haeckels Theorie in den „Vorarbeiten zum Anti-Dühring", Marx-Engels-Archiv, II, S. 402.

${ }^{8}$ Anti-Dühring, S. 64. 
Fetscher meint, daß Engels damit die "gesellschaftliche Bedingtheit" der Darwinschen Entdeckung vernachlässigt habe, ${ }^{1}$ die wir schon an Hand einer Äußerung von Marx erörtert haben. Dabei ist jedoch die Pointe des Engelsschen Gedankens übersehen: die Abhebung der wissenschaftlichen Richtigkeit der Theorie von ihrer Genese, eine Abhebung, die Fetscher dann übrigens selber vornimmt. ${ }^{2}$

Von da aus mußte Engels als ganz unhaltbar erscheinen, wenn man die Malthussche Bevölkerungstheorie, zusammen mit der ökonomischen Konkurrenz und Hobbes' bellum omnium contra omnes, erst in der Natur ,entdeckt”, nun wieder auf die menschliche Gesellschaft zurückübertrug und dann als deren ,natürliche" und somit ewig gültige Gesetze behauptete. In einem Brief an Lavrov hat Engels das I 875 auseinandergesetzt. ${ }^{3}$ Er betonte weiter, daß man Überproduktion von Gütern und periodische Handelskrisen, in denen diese Güter, ja sogar Produktivkräfte zerstört würden, in der gegenwärtigen ökonomischen Wirklichkeit nicht wohl durch die Formel „Kampf ums Dasein" wiedergeben könne, Kampf ums Dasein könne hier nur dies bedeuten, daß man durch eine sozialistische Revolution Produkte und Produktivkräfte dem zerstörerischen Kapitalismus aus den Händen reißen müsse $;{ }^{4}$ so sei überhaupt, setzte er hinzu, schon die Betrachtung der Geschichte als einer Geschichte von Klassenkämpfen der Auffassung, die Geschichte sei generell Kampf ums Dasein, weit überlegen und lasse deren „Seichtigkeit” erkennen. ${ }^{5} \mathrm{Zu}$ Malthus” Bevölkerungstheorie im besonderen wiederholte Engels 1865 in einem Brief an Lange - denselben Autor also, zu dessen Neomalthusianismus sich später Marx kritisch äußerte - einmal den Marxschen Vorwurf des Plagiats, sodann die eigene These aus dem frühen Essay „Umrisse zu einer Kritik der Nationalökonomie” ( 1844 ), ${ }^{6}$ daß die Bevölkerung nicht auf die Subsistenz-, sondern auf die Beschäftigungsmittel drücke. ${ }^{7}$

Mit dieser prinzipiellen Abgrenzung stand nicht in Widerspruch,

1 I. Fetscher, Von der Philosophie... (s.o. S. 433, Anm. I), S. 42 Anm. I.

${ }^{2}$ Fetschers Einwand wäre erst dann schlüssig, wenn seine Wiedergabe von Engels richtig wäre: er schreibt, nach Engels habe Darwin „keine Malthussche Brille” für seine Entdekkung gebraucht, bei Engels heißt es jedoch allgemein, „man” brauche diese Brille zur Wahrnehmung des Phänomens nicht, was für die damalige Zeit nicht weniger richtig ist als für die heutige.

${ }^{3}$ Engels an Lavtov, a.a.O., S. 357. Vgl. J. Schaxel, Darwinismus und Marxismus (s.o. S. 434, Anm. I), S. 487 f.

"Engels an Lavrov, a.a.O., S. 358 und - eine Zusammenfassung dieser Briefstelle - Dial. u. Natur, S. I9I.

- Engels an Lavrov, a.a.O., S. 358.

${ }^{6}$ Werke, Bd. I, Berlin I96r, S. 519.

"Engels an Lange, 29. März I865, Ausgewählte Briefe, S. 203. Engels äußert sich in diesem Brief zur x. Auflage der „Arbeiterfrage” (1865). 
sondern stimmte überein, wenn Engels ein Teilphänomen der gegenwärtigen - aber als solche eben aufzuhebenden - Wirklichkeit als Kampf ums Dasein beschrieb: die Konkurrenz der Kapitalisten untereinander. ${ }^{1}$ Er tat das, damit Marx’ Bemerkung im „Kapital” aufnehmend, in einer farbigen Schilderung im „Anti-Dühring” und machte dabei zugleich den Unterschied zur Natur deutlich. Nicht nur einzelne Kapitalisten, sondern darüber hinaus ganze Kapitalistengruppen und -klassen der verschiedenen Industrienationen stehen in einem Kampf auf Leben und Tod, der über den ganzen Globus hinweg geführt wird. In ihm entscheiden nicht mehr körperliche oder intellektuelle Fähigkeiten wie in der organischen Natur oder in der Ur- und Frühzeit des Menschen, sondern Standorte, Ressourcen und Produktivkräfte des eigenen Landes und der eroberten Kolonien. ${ }^{2}$ Aber gerade weil hier eine gewisse Analogie zur Natur zu beobachten sei, schrieb Engels in seinem schon erwähnten Brief an Lange, sei es eben „die höchste Blamage" für die bürgerliche Gesellschaft, den Menschen noch immer im Zustand tierischen Daseins gelassen zu haben $;^{3}$ und später notierte er in „Dialektik und Natur”, Darwin habe mit seinem Buch - unbewußt - eine „bittere Satire” auf die eigene Gesellschaft geschrieben. ${ }^{4}$ Damit meinte Engels vor allem, daß unter den Bedingungen der anarchischen Konkurrenz die Bewegungsgesetze der gesellschaftlichen Produktion nicht durchschaut, geschweige denn beherrscht werden; dazu werde, heißt es im "Anti-Dühring", den Menschen erst die sozialistische Revolution führen, und so scheide der Mensch erst in diesem Augenblick ,endgültig aus dem Tierreich". ${ }^{5}$

Nimmt man an dieser Stelle einmal Marx und Engels zusammen, so fällt auf, daß ihre Polemik gegen den Sozialdarwinismus nur eine Polemik gegen eine bestimmte Interpretation von Vergangenheit und Gegenwart ist. Die eigentliche Virulenz des Sozialdarwinismus, die er als zukunftsgestaltendes gesellschaftlich-politisches Programm gewinnen sollte, scheinen sie nicht geahnt zu haben, obwohl doch auch schon bei Darwin z.B. von bewußter Gattenwahl die Rede war. ${ }^{6}$

${ }^{1}$ Genau so später A. Pannekoek, Marxismus und Darwinismus (s.o. S. 434, Anm. I), S. 22.

2 Anti-Dühring, S. 254 f. L. Woltmann, Die Darwinsche Theorie und der Sozialismus (s.o. S. 434, Anm. I), interpretiert diese Stelle unzureichend einseitig auf das ,außermenschliche und technische Resultat" der Vervollkommnung der Maschine als gesellschaftlicher und damit „vom individuellen Menschen losgelösten" Produktivkraft (S. 30). Vgl. auch P. Lafargue, Der wirthschaftliche Materialismus (s.o. S. 454, Anm. 6), S. $25,28$.

3 Engels an Lange, a.a.O., S. 202 f.

'Dial. u. Natur, S. 252.

- Anti-Dühring, S. 264 .

- Abstammung d. Menschen, II, S. 355 f.; vgl. I, S. I46 ff. über die Auswirkungen der Zivilisation auf die natürliche Zuchtwahl. 
Ganz ähnlich scheint für Engels Darwins Selektionstheorie zu weit außerhalb des eigenen Vorstellungshorizontes gelegen zu haben. Er sah den Selektionsmechanismus nicht völlig von außen auf die mehr oder minder angepaßten Arten wirken, sondern sprach statt von Angepaßtsein wie Lamarck von "Anpassungsfähigkeit" 1 - was die Biologie als „funktionelle" Anpassung bezeichnet - und nannte die (aktiv verstandene) „Anpassung" eine „unbewußte Zwecktätigkeit”. ${ }^{2}$ Er blieb auch darin der Lamarckschen Tradition treu, daß er Vererbung erworbener Eigenschaften annahm, ${ }^{3}$ die Annahme also betonte, die der Neodarwinismus im Fortgang der Forschung aus der Theorie ausscheiden sollte. Daß diese Einordnung von Engels richtig ist, zeigt sich, wenn wir überlegen, daß Lamarck mit seiner „Philosophie zoologique" (1809) noch die alte teleologische Naturphilosophie repräsentiert; Engels jedoch urteilt über die Naturphilosophie, daß sie ein Gesamtbild des Naturzusammenhangs nur dadurch hätte geben können, daß sie die vielen noch offenen Lücken durch Spekulation ausfüllte, während heute der Gesamtzusammenhang am empirischen Material selbst zu gewinnen sei. ${ }^{4}$ Die Teleologie wird also nicht als Differenzpunkt herausgestellt. Das widerspricht nicht dem eingangs zitierten Engelsschen Urteil, Darwin habe die Teleologie erledigt, da er damit, wie wir zeigen konnten, die Physikotheologie des 17. und I8. Jahrhunderts meinte.

Damit interpretierte Engels den Verlauf der Evolution anders als Darwin. Diesem wurde es fraglich, was innerhalb der Evolution als Fortschritt, was als Rückschritt zu bezeichnen sei. ${ }^{5}$ Engels dagegen

1 Dial. u. Natur, S. 283 . Hervorhebung von mir.

2 Anti-Dühring, S. 66, vgl. Vorarbeiten zum „Anti-Dühring”, S. 402. Schon die Reaktion von Protoplasma ist für Engels „im Keime” eine „planmäßige Handlungsweise” (Der Anteil der Arbeit an der Menschwerdung des Affen, in: Dialektik der Natur, Werke, Bd. 20, S. 452 ).

"Dial. u. Natur, S. 225 und der ganze Essay „Der Anteil der Arbeit an der Menschwerdung des Affen", Werke, Bd. 20, S. 444-455. Dazu Darwin: die Vererbungsgesetze sind noch so gut wie unbekannt (Entstehung d. Arten, S. 24, I 81, 218 ; vgl. aber schon Darwins Beobachtungen über das Wiederauftauchen rezessiver Merkmale, S. 174, Ios ff.). Gebrauch und Nichtgebrauch unterstützen die natürliche Zuchtwahl, ihre Wirkungen vererben sich (S. 22, x 50 f., 228, 255; Abstammung d. Menschen, I, S. 100 ff.).

4 Ludwig Feuerbach und der Ausgang ...., S. 295.

s Darwin hielt es für schwierig anzugeben, was "größere Vollkommenheit" eines Lebewesens sei (Entst. d. Arten, S. 137 f., 516). Wenn damit besseres Angepaßtsein an die Lebensbedingungen durch Differenzierung der Organisation gemeint sei, dann könne dies, müsse aber nicht durch natürliche Zuchtwahl erreicht werden (145). Immerhin hielt Darwin Differenzierung und Spezialisierung der Organisation für den besten Maßstab für "Vollkommenheit” (239). Die Frage, ob die Evolution im Ganzen Fortschritt oder Rückschritt gebracht habe, ließ er als sehr "verwickelt” in der Schwebe (414). 
faßte es anders auf: jeder Fortschritt sei zugleich ein Rückschritt, da er durch die Fixierung einer Möglichkeit viele andere ausschließe.'Auch war für Engels, bei aller Ablehnung einer Zweckhaftigkeit im Verhältnis der Arten zueinander, der Verlauf der Evolution im Ganzen durchaus zielgerichtet: es liege im Wesen der Materie, denkende Wesen aus sich zu entwickeln. ${ }^{2}$ In diesen teleologischen - und damit inkonsequenten $^{3}$ - Materialismus hat Engels sogar die Wärmetodtheorie integriert: zwar werde die Sonne immer mehr erkalten, das Leben auf der Erde also allmählich aussterben und die Erde schließlich wie die anderen Planeten in die Sonne hineinfallen; und ebenso werde es früher oder später den anderen Planetensystemen ergehen. ${ }^{4}$ Aber dies sei nicht das endgültige Schicksal des Kosmos, sondern eines Tages werde die Materie ihre Bewegung wieder differenzieren, ${ }^{\mathbf{5}}$ und schließlich werde sie auch wieder irgendwo ,ihre höchste Blüte, den denkenden Geist . . . erzeugen" ${ }^{6}$ Grundlage dieses ewigen Kreislaufprozesses 7 ist für Engels die sich bewegende Materie, „unerschaffbar und also ihre eigne Endursache", 8 "causa finalis". 9

So steht im Zentrum der Engelsschen Naturphilosophie das Ziel, nicht wie Hegel die Natur als „Außersichsein des Geistes” aufzufassen, sondern den Geist in den einheitlichen Gesamtprozeß des Kosmos hineinzunehmen ;0 die Geschichte des Menschen erscheint nur noch „als Entwicklungsprozeß selbstbewußter Organismen von der Geschichte der Natur verschieden".11 Das bedeutete für Engels den Primat der anorganischen Natur vor der organischen. In diesem Sinne warf er im "Anti-Dühring" der Naturwissenschaft vor, noch immer nicht organisches Leben auf chemischem Wege erzeugt zu haben. Auch Darwin erörtere den Ursprung des Lebens nicht, sondern leite nur innerhalb der Grenzen der organischen Natur eine Art von der anderen her. ${ }^{12}$ Den pessimistischen Hinweis von Helmholtz, daß es

1 Dial. u. Natur, S. 283.

2 S. 202.

${ }^{3}$ Fetscher, a.a.O., S. 49 f.; vgl. A. Schmidt, a.a.O., S. 48 .

- Dial. u. Natur, S. 252.

S. 253.

S. 255 .

${ }^{7}$ S. 175 .

S. 233 .

S. I55.

${ }^{10}$ So G. Mayer, Friedrich Engels, II, S. 302.

${ }^{11}$ Dial. u. Natur, S. x 59. „Natur und Mensch schließen sich bei Engels nicht primär [scil. wie bei Marx] vermittels historischer Praxis zusammen; der Mensch erscheint nur als Evolutionsprodukt und passiver Spiegel des Naturprozesses, nicht aber als Produktivkraft", A. Schmidt, a a.O., S. 45 .

12 Anti-Dühring, S. 67. Darwin klammerte diese Frage in der Tat entschieden aus (Entst. d. Arten, S. 278, 559). 
bisher noch nicht gelungen sei, Leben künstlich zu erzeugen, schob Engels mit der Bemerkung beiseite, man müsse nur erst die chemische Zusammensetzung des Eiweißes kennen, dann werde man es auch künstlich erzeugen können; damit hat er ja durchaus recht behalten, allerdings meinte er ganz irrtümlich, doch dem damaligen Wissensstand entsprechend, daß künstlich erzeugtes Eiweiß auch Lebensreaktionen zeigen werde. ${ }^{1}$ Aber eben: das war überhaupt mehr als Zutrauen in den Fortschritt der Forschung: anders als für Marx, der zwar aufmerksam, aber doch wie jede andere wissenschaftliche Frage die naturwissenschaftlichen Untersuchungen über den Ursprung des Lebens verfolgte, ${ }^{2}$ hing für Engels nicht weniger daran als seine "ganze Weltanschauung". ${ }^{3}$

Engels hat dann im einzelnen gezeigt, wie er sich die Entwicklung des denkenden Geistes auf der Erde vorstellte. Dabei machte er zwei Grundannahmen:

r. der Mensch hat sich nicht an mehreren Stellen der Erde, sondern nur an einer einzigen von den Affen gesondert; 4

2. am Anfang der menschlichen Geschichte herrschte nicht der Kampf aller gegen alle ${ }^{5}$ der Urmensch lebte gesellig, denn nur im größeren Verband war ihm die Erhebung über das Tier möglich. ${ }^{6}$

Auf Grund dieser zweiten Annahme hat Engels in der 4. Auflage seiner Schrift „Der Ursprung der Familie, des Privateigentums und des Staats" (189r) mit Entschiedenheit einen Urzustand allgemeiner Promiskuität gegen Angriffe von Kritikern verteidigt. ${ }^{7}$ Er ging dabei von der Beobachtung aus, daß bei den höheren Tieren Eifersucht und Zugehörigkeitsgefühl zur Horde sich ausschließen. ${ }^{8}$ Lebte nun der affenähnliche Vorfahr des Menschen gesellig, dann muß er frei von

${ }^{1}$ Dial. u. Natur, S. I $7^{8} \mathrm{ff}$.

${ }^{2}$ Marx an Engels, 18. Okt. 1868, III/4, S. 1 14.

${ }^{3}$ G. Mayer, Friedrich Engels, II, S. 317.

${ }^{4}$ Engels an Marx, 16. Nov. 1864, III/3, S. 203 f.

${ }^{5}$ Engels an Lavrov, 12.-17. Nov. I875, a.a.O., S. 359.

' Der Ursprung der Familie, des Privateigentums und des Staats, in: K. Marx/F. Engels, Ausgewählte Schriften, Berlin 1955, II, S. I 83 f.; der Mensch ist „das geselligste aller Tiere", Der Anteil der Arbeit a.a.O., S. 446, vgl. S. 444. Nach Darwin lebte der Urmensch "wahrscheinlich gesellig", Abst. d. Menschen, I, S. I 34, vgl. S. I 40 f.

${ }^{7}$ Der Ursprung der Familie (s. vor. Anm.), S. I 8 I ff. Daß auch Darwin zu den Bestreitern gehörte (Abst. d. Menschen, II, S. 3 I 6 ff.), erwähnte Engels nicht. Als Kautsky aber in seiner Arbeit „Die Entstehung der Ehe und Familie" (Kosmos, I 2, I 882/83, S. 190 ff., 256 ff., 329 ff.) die Urpromiskuität ablehnte und dabei u.a. auf Darwins Argumente verwies (S. I 99 ff.), erwiderte ihm Engels, Darwin sei für die Urgeschichte „ebenso wenig Autorität, wie in der Oekonomie, woher er seinen Malthusianismus” importiere (Brief vom ro. Febr. I 883, in: F. Engels, Briefwechsel mit Karl Kautsky, 2. Aufl., Wien 1955, S. 72).

${ }^{8}$ Der Ursprung der Familie, S. $182 \mathrm{f}$. 
Eifersucht gewesen sein. ${ }^{1}$ Einzeln oder in Einzelpaarung lebende Menschenaffen wie der Gorilla und der Schimpanse sind nach Engels niedergehende oder aussterbende Seitenlinien. ${ }^{2}$

Damit aber geriet Engels in Widerspruch zu anderen Ausführungen seines Buches. Im Anschluß an Morgan hatte er für die Frühgeschichte des Menschen folgendes darwinistische Schema aufgestellt. Die Gesellschaft dieser Zeit kannte keine Klassen, sie entwickelte sich also nicht in dialektischen Sprüngen, die nach dem historischen Materialismus die gesellschaftliche Entwicklung seit der Antike kennzeichnen. Daß die Urgesellschaft trotzdem nicht stationär war, lag an der natürlichen Zuchtwahl, die Fortschritt ermöglichte: in einer schrittweisen Einschränkung der Promiskuität und damit Verminderung der Möglichkeit von Inzucht etlangten immer die Stämme im Kampf ums Dasein das Übergewicht über ihre Nachbarn, die in ihrer Familienform der Einzelpaarung am nächsten gekommen waren. ${ }^{3}$ Was also für das Mensch-Tier-Übergangsfeld als der entscheidende Vorteil des Menschen gegenüber dem Tier gepriesen wird - die Promiskuität -, wird für den weiteren Verlauf der Frühgeschichte als der entscheidende Nachteil im Kampf ums Dasein zwischen den verschiedenen Horden und Stämmen hingestellt. Doch ist deutlich, daß es sich hier nicht um wissenschaftliche Erkenntnis handelt, sondern um Hypothese und Postulat, gefolgert aus eigenen Axiomen. ${ }^{4}$

Ausführlicher und nicht durch eine Polemik herausgefordert hat Engels seine Vorstellung von der Abstammungsgeschichte des Menschen in dem Essay „Der Anteil der Arbeit an der Menschwerdung des Affen" (1 876) dargelegt. ${ }^{5}$ Hier zeichnet er folgendes Bild. Mit dem aufrechten Gang ,war der entscheidende Schritt getan für den Übergang vom Affen zum Menschen".6 Jetzt konnte sich die Hand endgültig vom Fuß differenzieren. ${ }^{7}$ Von anfänglich einfachen lernten die Urmenschen ihre Hand an immer kompliziertere Verrichtungen anzupassen, „und die damit erworbene größere Biegsamkeit vererbte und vermehrte sich von Geschlecht zu Geschlecht. So ist die Hand nicht nur das Organ der Arbeit, sie ist auch ihr Produkt". ${ }^{8}$ Nach dem Gesetz „der Korrelation des Wachstums, wie Darwin es genannt hat”,

${ }^{1} \mathrm{~S} .183 \mathrm{f}$.

S. 184 .

"Vgl. meinen Aufsatz „Die Rezeption Lewis H. Morgans durch Marx und Engels", in: Sxculum, I 5, I964, S. I68 f., für Morgan S. 161.

4 S. 175 und Anm. 299.

${ }^{5}$ In Werke, Bd. 2o, S. 444-455. Nach der redaktionellen Note S. 663 ursprünglich als Teil einer größeren Arbeit gedacht und dann, als diese nicht fertig wurde, von Engels mit diesem Titel versehen. Eine Zusammenfassung des Inhalts findet sich in Dial. u. Natur, S. $250 \mathrm{f}$.

- S. 444. 7. S45. ${ }^{8}$ Ebenda. 
hatte die Entwicklung der Hand Rückwirkungen auf den übrigen Organismus; worin sie bestanden, muß nach Engels jedoch noch untersucht werden. ${ }^{1}$ Ein Punkt aber ist sicher: Geselligkeit und zunehmende Herrschaft über die Natur führte die Menschen allmählich „dahin, daß sie einander etwas zu sagen hatten", d.h. Engels will die „Entstehung der Sprache aus und mit der Arbeit" erklären. ${ }^{2}$ Erst zuletzt entwickelte sich das menschliche Hirn - zuerst durch die Arbeit, „nach und dann mit ihr” durch die Sprache. ${ }^{3}$ Die „Entwicklung des Gehirns und seiner dienstbaren Sinne, des sich mehr und mehr klärenden Bewußtseins, Abstraktions- und Schlußvermögens" wirkte dann wieder zurück ,auf Arbeit und Sprache".4 Jedoch ungeachtet dieser Reihenfolge entstand schon bald die Neigung der Menschen, ,ihr Tun aus ihrem Denken zu erklären statt aus ihren Bedürfnissen (die dabei allerdings im Kopf sich widerspiegeln, zum Bewußtsein kommen)", 5 entstand also die verderbliche Neigung zum Idealismus, die erst heute durch die Ergebnisse der Naturwissenschaft allmählich verschwindet. ${ }^{6}$

Der Übergang vom Tier zum Menschen wird also erklärt aus der Steigerung und Differenzierung der materiellen Bedürfnisse und der anschließenden Vererbung der somatischen Erwerbungen, wobei eine genaue Reihenfolge der Entwicklung aufgestellt wird: Hand (Arbeit) - übriger Organismus - Sprache - Kopf; die geistige Tätigkeit wird reduziert auf Widerspiegelung der Bedürfnisse und technische Intelligenz. Dies reduzierte Verständnis der Geistigkeit kennzeichnet jedoch nicht nur Engels' Hypothese für das Mensch-Tier-Übergangsfeld; die den Menschen auszeichnende Differenz zu seinen Produkten, seinen Handlungen und zur Gesellschaft verschwinden bei Engels ganz allgemein, wie folgende Bestimmungen des Unterschiedes zwischen Mensch und Tier verdeutlichen mögen. Die Tiere bringen es höchstens zum Sammeln, ,während die Menschen produzieren". ${ }^{7}$ - Der Mensch trat in dem Augenblick aus dem Tierreich, als er nicht nur unmittelbare Subsistenzmittel produzierte, sondern die Zeit fand zur Produktion von Produktionsmitteln. ${ }^{8}-$ Die Tiere adaptieren sich ihrer Umwelt, der Mensch schafft sich seine Existenzverhältnisse. ${ }^{9}-$ „Das Tier benutzt die äußere Natur bloß ... der Mensch macht sie durch seine Änderungen seinen Zwecken dienstbar, beherrscht sie."10

1S. 446. Der Hinweis trifft nicht zu; Darwin spricht von „korrelativen Variationen” (Entst. d. Arten, S. 23, I59; Abst. d. Menschen, I, S. I I 2 f.), womit etwas anderes gemeint ist.

S. 446 f. $\quad$ S. 447 . S. 448.

${ }^{5}$ S. 450 f., wörtliches Zitat S. $45 \mathrm{I}$.

S. 453 .

${ }^{7}$ Brief an Lavrov, I 2.-17. Nov. I875, a.a.O., S. 357.

${ }^{8}$ Anti-Dühring, S. ז80. 'Dial. u. Natur, S. I 75 f.

${ }^{10}$ Der Anteil der Arbeit, S. 452. 
Fassen wir zusammen. Marx und Engels haben Darwins „Entstehung der Arten" freudig begrüßt und sich dann immer wieder damit beschäftigt. Sie haben dabei eine für Nichtnaturwissenschaftler erstaunliche Vielfalt der Aspekte entdeckt, der gegenüber die Schranken ihres Verständnisses nicht ins Gewicht fallen. Ihre Interpretationen gehen jedoch, trotz sehr vieler Berührungspunkte und gegenseitiger Anregung, nicht in die gleiche Richtung. Diese Differenz wird dort am deutlichsten, wo Marx und Engels den Einfluß Darwins in die eigenen Auffassungen hineingenommen und verarbeitet haben.

Marx hat im „Kapital” das Darwinsche Naturmodell als Ausgangspunkt für einen großen Geschichtsentwurf benutzt, der als Ortsbestimmung der Gegenwart und der jüngeren Vergangenheit dienen sollte. Danach hat sich der Mensch auf dem Wege seiner bisherigen Geschichte weit und unwiderruflich von ,natürlichen" Lebensbedingungen entfernt. Die gegenwärtige Produktionsweise, deren revolutionäre technische Basis alle herkömmlichen gesellschaftlichen Verhältnisse radikal zerstört hat und auch keine dauerhafte neue Ordnung zustandekommen läßt, sondern nach den Exploitationsbedürfnissen des Kapitals ungeheure Menschenmassen umherschleudert, hat den Menschen in einen Degenerationsprozeß gerissen, dem er nur entrinnen kann, wenn es ihm gelingt, den gesellschaftlichen Mechanismus unter Kontrolle $\mathrm{zu}$ bringen und seine Lebensbedingungen völlig rational zu gestalten. Die realgeschichtliche Aufeinanderfolge von Produktionsweisen sieht Marx andererseits auch direkt in Analogie zum Evolutionsprozeß der Natur, in dem sich eine Art aus der anderen entwickelt. Hier ergibt sich eine gewisse Annäherung an den Evolutionismus des späten Engels, die jedoch an der positiv-endlichen Anthropologie von Marx ihre Grenze findet. Vom Aspekt des Verhältnisses von Individuum und Gattung aus jedoch scheidet der Mensch erst mit der klassenlosen Gesellschaft aus der Natur, ist also die bisherige Geschichte für Marx nur „Vorgeschichte”.

Fand Marx bei Darwin so die naturhistorische Grundlegung für seinen Geschichtsentwurf, begab sich Engels auf das von Darwin selbst bearbeitete Gebiet der Abstammungs- und Frühgeschichte des Menschen. In „Der Anteil der Arbeit an der Menschwerdung des Affen" entwickelt er den Übergang vom Tier zum Menschen vollständig aus materiellen Bedürfnissen und anschließender Vererbung der somatischen Erwerbungen; die geistige Tätigkeit hat dabei nur die Funktion, die Bedürfnisse widerzuspiegeln und die Mittel zu ihrer Befriedigung zu erfinden, wird also reduziert auf technische Intelligenz. Im „Ursprung der Familie, des Privateigentums und des Staats” 
wird die allmähliche Beschränkung der Inzucht zum Bewegungsprinzip der Frühgeschichte erklärt; die kräftigeren und intelligenteren Stämme, nämlich die Stämme mit einem Weniger an Inzucht, setzten sich danach im Kampf ums Dasein durch. Schließlich hat Engels in der „Naturdialektik”, nicht so zentral im „Anti-Dühring”, die Evolution des Menschen eingebettet in die umfassendere Evolution der Natur. Nur durch sein Selbstbewußtsein ist der menschliche von den übrigen Organismen unterschieden. Jedoch hat er sich mit Notwendigkeit aus der übrigen Natur entwickelt. Diese Aufstellungen haben mit dem historischen Materialismus von Marx nichts mehr zu tun. Sie deuten vielmehr in die Richtung des Materialismus von Karl Kautsky.

\section{$A N H A N G$}

DER BRIEFWECHSEL ZWISCHEN MARX UND DARWIN

Die Marx- und Darwinliteratur hat eine, allerdings ungenaue Kenntnis von zwei Briefen Darwins an Marx aus den Jahren i 873 und i 880, in denen er auf Schreiben antwortete, die Marx an ihn gerichtet hatte. Wir geben sie hier nach den Originalen wieder, die sich im MarxEngels-Nachlaß des Internationalen Instituts für Sozialgeschichte, Amsterdam, befinden. ${ }^{1}$

Der erste Brief wurde 1897 von Aveling, dem Gatten der dritten Marx-Tochter Eleanor, in seinem Aufsatz „Charles Darwin and Karl Marx" veröffentlicht, ${ }^{2}$ von dem eine deutsche Übersetzung in der Neven Zeit ${ }^{3}$ und eine französische in Le Devenir Social ${ }^{4}$ erschien. Avelings Wiedergabe ist nicht ganz korrekt; die Abweichungen werden in den Textnoten mitgeteilt. Der Brief lautet:

Oct. I. 73

Down,
Beckenham, Kent.

Dear Sir

I thank you for the honour which you have done me by sending me your great work on Capital; and I heartily wish that I was ${ }^{b}$

a Ortsangabe vorgedruckt $\quad$ b were Aveling

\footnotetext{
1 Sie sind dort katalogisiert unter der Nummer D. II. I 2-1/2. Dem Institut möchte ich auch an dieser Stelle danken, daß es mir Einsichtnahme und Abdruck der Briefe gestattete.

${ }^{2}$ Charles Darwin and Karl Marx, in: The New Century Review, I, I897, S. 232-243, 321-327; der Brief S. 243, im Sonderdruck des Aufsatzes S. Ix.

${ }^{3}$ Neue Zeit, 15/2, $1896 / 97$, S. 745-757; der Brief S. 753.

${ }^{4}$ Le Devenir Social, 3, I 897, S. 346-363; der Brief S. 357.
} 
more worthy to receive it, by understanding more of the deep and important subject of political economy. Though our studies have been so different, I believe that we both Earnestly desire the extension of knowledge, ${ }^{a}$ and that ${ }^{b, c}$ this in the long rund is sure to add to the happiness of Mankind.e

I remain Dearf Sir

Yours faithfully

Charles Darwin

Der zweite Brief hat folgenden Wortlaut:

\section{Private}

Oct. I 3. 1880

\section{Down, Beckenham, Kent.}

$\left(\begin{array}{c}\text { Railway Station } \\ \text { Orpington. S.E.R. }\end{array}\right) \mathrm{g}$

\section{Dear Sir}

I am much obliged for your kind letter and the enclosure. - The publication in any form of your remarks on my writings really requires no consent on my part, and it would ${ }^{\text {h }}$ be ridiculous in me to give consent to what requires none. - I sh[ould] prefer the Part or Volume not to be dedicated to me (though I thank you for the intended honour) as this implies to a certain extent my approval of the general publication, about which I know nothing. - Moreover though I am a strong advocate for free thought on all subjects, yet it appears to me (whether rightly or wrongly) that direct arguments against christianity and theism produce hardly any effect on the public; and freedom of thought is best promoted by the graduali illumination of men's ${ }^{j}$ minds, which follows from the advance of science. It has, therefore, been always my object to avoid writing on religion, and I have confined myself to science. I may, however, have been unduly biassed by the pain which it would give some members of my family, if I aided in any way direct attacks on religion. - 
I am sorry to refuse you any request, but I am old and have very little strength, and looking over proof-sheets (as I know by present experience) fatigues me much. -

I remain Dear Sir yours faithfully

Ch. Darwin

Der zweite Brief ist auf einem ziemlichen Umweg und ungenau in die Literatur gelangt. Er wurde 193 I vom Moskauer Marx-EngelsInstitut der deutschen Zeitschrift Der rote Aufbau zur Verfügung gestellt - das Moskauer Institut besaß eine Fotokopie des damals im SPD-Archiv befindlichen Originals -, und die Zeitschrift druckte ihn in deutscher Übersetzung ab. ${ }^{1}$ Diese Übersetzung wurde von der Zeitung Welt am Abend, die wie Der rote Aufbau zum sogenannten „Münzenberg-Konzern” gehörte, nachgedruckt. Nun wurde die Londoner Times auf ihn aufmerksam; sie brachte ihn, rückübersetzt ins Englische, am ı. Mai 193 I. $^{2}$ Bei dieser zweimaligen Weitergabe wurde nicht exakt verfahren: hatte Der rote Aufbau nur geschrieben, das Moskauer Institut habe ihm den Brief „zur Verfügung gestellt”, also keine Angabe über das Original gemacht, so setzte die Times hin$\mathrm{zu}, \mathrm{da} ß$ sich der Brief im Moskauer Institut befände. ${ }^{3}$ Die Rückübersetzung der Times wurde nun wiederum von der englischen kommunistischen Zeitschrift The Labour Montbly nachgedruckt mit folgender Vorbemerkung: „The Text ... was published recently for the first time by the Marx-Engels Institute in Moscow, and a copy appeared in the ,Times' of May, 18, 1931."4 Daraus konnte geschlossen werden, daß hier der originale Text wiedergegeben werde; diese Annahme machte Keith in seinem Buch über Darwin (1955) und druckte den Text noch einmal - mit einer geringfügigen Auslassung - nach. ${ }^{5}$ Es lohnt natürlich nicht, eine der Fassungen der Times, von The Labour Montbly und Keith wiederzugeben. Sie weichen nur geringfügig voneinander, jedoch überaus häufig vom Original $\mathbf{a b} .^{\mathbf{6}}$

Wir wenden uns nun der Analyse der Briefe zu. Zum ersten ist nur wenig zu bemerken. Marx hatte Darwin die zweite deutsche Auflage

${ }^{1}$ Ein unbekannter Brief Darwins, in: Der tote Aufbau, 4, 1931, S. 357.

2 The Times, 18. Mai I93I, S. II.

A.a.O.

4 The Labour Monthly. A Magazine of International Labour, 1 3, I93 I, S. 702.

${ }^{5}$ A. Keith, Darwin revalued, London I955, S. 234.

- Mehr als $30 \mathrm{mal}$, fast immer stilistisch oder in der Wortwahl. 
des „Kapital” zugesandt. ${ }^{1}$ Die Antwort Darwins ist nicht nur, wie die Moskauer Marx-Chronik schreibt, eine „höfliche Empfangsbestätigung" ${ }^{2}$ mit Prenant wird man hinzusetzen müssen, daß sie „ausweichend" ist, wobei man zu beachten hat, daß Darwin gewöhnlich auf die Zusendung eines Buches „mehr als höflich” antwortete. ${ }^{3}$

Schwierigkeiten bereitet dagegen der zweite Brief. Klar ist zunächst nur, daß mit „the Part or Volume” der 2. Band des „Kapital" gemeint ist, ${ }^{4}$ den Marx dann nicht mehr bis zur Endredaktion brachte. Darwin lehnt Marx' Bitte um Zustimmung zur Widmung ab mit der Begründung, die Widmung würde auf ein gewisses Einverständnis mit dem ganzen Werk schließen lassen, über das er jedoch nichts wisse. Zum Verständnis dieser Begründung muß man sich vor Augen halten, daß im 19. Jahrhundert noch nicht die Unsitte eingerissen war, daß ein Autor jemandem ein Buch widmet, ohne ihn vorher um sein Einverständnis gefragt zu haben; eine Widmung hatte damals also einiges Gewicht. ${ }^{5} \mathrm{Da}$ Darwin es ablehnt, von Marx etwas zu lesen, scheint seine Zurückhaltung ganz erklärlich.

Worauf bezieht sich nun der folgende Passus, in dem Darwin seine Haltung zur Religion auseinandersetzt? Der Darwin-Biograph v. Wyss nimmt an, Marx habe Darwin ,für eine antireligiöse Kampagne zu gewinnen" versucht. ${ }^{6}$ Das ist jedoch ganz unwahrscheinlich. Nicht nur, daß Marx bereits aus den Schlußworten der „Entstehung der Arten" das Bemühen Darwins kannte, seine wissenschaftlichen Untersuchungen und Thesen aus der religiösen Diskussion herauszuhalten;? Marx war seinerseits areligiös, desinteressiert ,an Religion in jeder Form, auch am Kampf gegen die Religion". 8 So bleibt nichts anderes übrig, als diesen Passus auf die vorangehende Ablehnung von Marx'

'So richtig E. Aveling, a.a.O., S. 243. Die Bandausgabe der 2. Auflage des „Kapital” erschien Mitte Mai 1873 (Karl Marx. Chronik seines Lebens in Einzeldaten, Moskau I 934, S. 343; im Folgenden zitiert: „Chronik”). Falsch dagegen „Der rote Aufbau” (s.o. S. 466, Anm. I), der von der französischen Úbersetzung (Paris 1 872-75) spricht (s. u.).

${ }^{2}$ Chronik, S. 345.

${ }^{3}$ M. Prenant, Darwin, Paris 1938, S. 47 (2. Aufl. 1946, S. 43).

- Chronik, S. 379. Autoren, die nur flüchtigen Einblick nahmen, konnten hier dadurch in Verwirrung geraten, daß nur der 1 . Band des „Kapital” eine Widmung (dem Gedächtnis Wilhelm Wolffs) trägt. Man vergleiche hier die vorsichtige Notiz von G. West in seiner vorzüglichen Darwin-Biographie: Charles Darwin, New Haven 1938, S. 324 mit den ebenso sicheren wie falschen Ausführungen von I. Berlin, Karl Marx. Sein Leben und sein Werk, München I959 (englische Erstausgabe London-New York-Toronto 1939), S. 261, der die beiden Darwin-Briefe einfach journalistisch zusammengewischt hat.

${ }^{5}$ Ich verdanke diesen Hinweis Herrn Prof. D. Helmut Gollwitzer.

- W. v. Wyss, Charles Darwin. Ein Forscherleben, Zürich-Stuttgart 1958, S. 277. Bei den folgenden Bemerkungen bin ich wiederum Herrn Prof. Gollwitzer zu Dank verpflichtet.

7 Entst. d. Arten, S. 559, 56 i f., 569.

${ }^{3}$ H. Gollwitzer, Die marxistische Religionskritik und der christliche Glaube, in: Marxismusstudien, IV, Tübingen 1962, S. 19 ff., 29 f., wörtliches Zitat S. 22. 
Bitte an Darwin, die Widmung anzunehmen, zu beziehen, wofür auch das anknüpfende „moreover" spricht: ${ }^{1}$ Darwin fügt als weiteren Grund für seine Ablehnung der Bitte an, auch den leisesten Anschein vermeiden zu wollen, militanten Atheismus zu unterstützen; er zeigt sich so in den bürgerlichen Vorstellungen seiner Zeit befangen - und für diese Befangenheit gibt es auch andere Belege -, indem er Sozialismus und öffentlichen Kampf gegen die Religion automatisch in Verbindung setzt. ${ }^{2}$ Dabei macht er weitreichende Zugeständnisse: er argumentiert nur mit der Erfolglosigkeit von Angriffen auf die Religion, stellt also nicht die Berechtigung des Atheismus in Frage, und gibt auch persönliche Rücksichtnahme zu; ${ }^{3}$ gleichzeitig hindert er Marx an einer möglichen Weitergabe oder publizistischen Auswertung seiner Äußerungen, indem er den Brief als „private” kennzeichnet.

Offen sind nun noch Anfang und Schluß des Briefes, d.h. die folgenden Fragen: I) was hat Marx seinem Brief an Darwin beigefügt? 2) sind Marx' Bemerkungen über Darwins Schriften bereits veröffentlicht, oder plant Marx sie erst? 3) was ist mit den „proof-sheets” gemeint?

Die Zeitschrift Der rote Aufbau schickt dem Brief eine Vorbemerkung vorauf, in der es heißt, Marx habe Darwin gebeten, ,in der Korrektur der englischen Ausgabe des ,Kapitals' die Stellen durchzusehen (Kap. XII und XIII), wo Marx auf die Evolutionslehre eingeht", und diese Kapitel habe Marx Darwin dann widmen wollen. Weiter wird auf den früheren Brief von I 873 verwiesen, mit dem Darwin „für die Zusendung eines Exemplares der französischen Übersetzung des ,Kapitals"' gedankt habe. " Diese Angaben sind bis hin zu The Labour Montbly nachgedruckt worden und auch in das Buch von Prenant über Darwin ${ }^{5}$ eingegangen, sie sind aber in drei Punkten offenkundig falsch. Mit der Widmung ist, wie schon dargelegt, etwas anderes gemeint; die französische Übersetzung des „Kapital” erschien von 1872 bis 1875 in Lieferungen, war also I 873 noch unabgeschlossen; die erste englische Ausgabe, auf die sich der zweite Brief beziehen soll, erschien erst I 886 nach Marx' Tod. So wird das Zutrauen in den

\footnotetext{
I So Chronik, S. 379 .

2 Gröber die 2. Auflage der „Bol’šaja Sovetskaja Enciklopedija”, Artikel „Darvin”, Bd. I3, 1952: Darwin „fürchtete, seinen Namen mit dem Namen des großen Revolutionärs zu verbinden" (369).

${ }^{3}$ Dieser Punkt wird in dem Aufsatz von E. Kolman „Über den sogenannten 'Agnostizismus' Darwins” ausgeschlachtet, den „Der rote Aufbau” der Veröffentlichung des Briefes beifuigte (a.a.O., S. 358-359; englisch nachgedruckt von "The Labour Monthly", a.a.O., S. 703-705).

4 Der rote Aufbau, a.a.O.

5 S.o. S. 467 , Anm. 3.
} 
wichtigen Hinweis auf das I2. und I3. Kapitel des „Kapital” stark gemindert.

Gleichwohl gibt es keine andere Möglichkeit der Orientierung. Allerdings wird man den fraglichen Abschnitt nach vorne weiter fassen müssen, nämlich vom historischen Teil des 8 . bis zum I 3 . Kapitel; diese Partie ist, wie wir zeigten, in der Tat in einer Auseinandersetzung mit Darwin geschrieben. Man kann dann vermuten, daß Marx die Lieferung dieses Abschnitts aus der französischen Übersetzung dem Brief beigefügt und Darwin um eine Stellungnahme zu den hier aufgestellten Thesen über die Evolution des Menschen unter den Bedingungen der modernen Industriegesellschaft gebeten hat. Fragt man von da aus weiter, wozu Marx die erhoffte Zustimmung benutzen wollte, bieten sich zwei Möglichkeiten an. Entweder sollte sie die Grundlage für die Annahme der Widmung des 2. Bandes sein. Mit den „remarks on my writings" und vermutlich auch mit den „proof-sheets” wäre dann der beigefügte „Kapital”-Abschnitt gemeint. Oder Marx wollte sie für eine spezielle Publikation gegen den Sozialdarwinismus verwerten, der diesem dann das Recht bestritten hätte, sich auf Darwin zu berufen. Die fraglichen Sätze könnten dann entweder den vorliegenden Abschnitt oder die beabsichtigte Publikation meinen; im letzteren Falle hätte Marx Darwin um deren Durchsicht gebeten. Weiter als zu diesen Hypothesen kommt man ohne den anscheinend verlorengegangenen Brief von Marx an Darwin nicht. 